\title{
Discrete choice models with capacity constraints: an empirical analysis of the housing market of the greater Paris region
}

\author{
André de Palma* \\ Nathalie Picard ${ }^{\dagger}$ \\ Paul Waddell ${ }^{\ddagger}$
}

February 21, 2007

\begin{abstract}
Discrete choice models are based on the idea that each user can choose both freely and independently from other users in a given set of alternatives. But this is not the case in several situations. In particular, limitations and interactions can occur when the number of available products of one type is smaller than the total demand for this type. As a consequence, some individuals can be denied their preferred choice. We develop a methodology to address those constraints and we apply it to residential location choice, where our empirical data suggest that availability constraints may bias actual choices. The analysis provides some theoretical developments and elaborates an iterative procedure for estimating demand in the presence of capacity constraints. The empirical application relies on the location choice model developed and estimated in [6] for Ile de France (Paris region) and generalizes it to integrate capacity constraints.

Keywords: Residential location, constrained Logit, capacity constraints, sampling, Ilede-France.

JEL: C35, R21, R31.
\end{abstract}

\section{Introduction}

The choices that individual households make in the housing market produce aggregate outcomes that shape urban traffic conditions, patterns of poverty concentration and ethnic segregation, the quality of public schools, access to economic opportunities, and the decline and revitalization of neighborhoods - all of which are important, long-term, and related policy concerns. Efforts to model individual household residential location choices using discrete choice frameworks date at least to the pathbreaking work of [9], [11], [12], [13], and [14], among others. Over the past 25 years an extensive literature has examined household location choices in the housing market, significantly advancing the behavioral underpinnings and the methods used, and including efforts to represent the residential location choice as a dynamic process: see, e.g., [1].

\footnotetext{
${ }^{*}$ University of Cergy-Pontoise, THEMA, France and Ecole Nationale des Ponts et Chaussée, France. Member of the Institut universitaire de France. e-mail: andre.depalma@u-cergy.fr.

${ }^{\dagger}$ University of Cergy-Pontoise, THEMA, France and INED, France. e-mail: nathalie.picard@u-cergy.fr.

${ }^{\ddagger}$ University of Washington at Seattle, Department of Urban Design and Planning,Box 353055, Seattle, Washington 98195-3055, Phone: (206) 221-4161 Fax: (206) 685-9044, email: pwaddell@u.washington.edu, corresponding author.
} 
One important issue that has not yet received sufficient attention in the literature, and that is the central focus of the theoretical and empirical analyses presented in this paper, is the role of availability constraints in households' choices. In estimating location choice models by observing agents' choices among a set of alternatives, an implicit assumption is made that the alternatives are all available, as they would commonly be if the choice were made among standard (durable or non durable) commodities which are not in short supply. However, in the housing market and in many other market situations, such as air travel, limited availability is not at all uncommon. For example, a particular neighborhood may be highly desired, while few vacancies may be available to those searching in this area. Seats may be sold out on the particular flight itinerary a traveler wishes to choose. A standard assumption in economics is that prices adjust and clear the market, and therefore putting prices on the right hand side of the model is sufficient to address this concern. However, casual observation supported by data suggest that this assumption may be too strong in many market conditions, including the housing market. Various forms of friction make the housing market less than perfectly efficient. High transactions costs, attachments to social networks, non-trivial search costs, asymmetric information, low turnover in some locations, and sellers' willingness to withhold a property from the market rather than suffer a loss, among other factors, suggest that prices may not fully clear the market. These factors give rise to supply constraints (or sticky prices) in some locations. Although the phenomenon we analyze is plausible to find in many metropolitan housing markets, it is likely that certain characteristics increase the likelihood of finding excess demand in highly dense housing markets such as that in Paris. Its large population and international attractiveness as a tourist destination, coupled with the degree of preservation of the historical core of the city, mean that supply is not likely to be very elastic in the urban core. Moreover, vacancies may be structurally high due to speculation and given that a significant fraction of the housing stock is held by non-residents. It is certainly not difficult to imagine that housing in the most desirable locations of Paris might not be sufficient for supply to meet demand.

Whether prices actually do fully clear the housing market should be an empirical question rather than a strong assumption concerning the perfect competition hypothesis. If the assumption that prices clear the market is not valid, then it follows that coefficients estimated for discrete choice models in markets that experience some level of availability constraints will be biased, confounding the effect of the constraints with the agents' preferences. An important policy implication of this methodological concern is that if these constraint effects are not corrected for in estimation of a choice model, predicted shifts in demand in response to an exogenous change (such as the change in accessibility due to major transportation investments) would also be biased, leading to potentially misleading conclusions regarding the relative costs and benefits of alternative policy choices. A related concern is that housing prices are jointly determined with location choices in constrained housing markets due to the role of prices in clearing the market, even if they do not completely clear it. It is therefore necessary to address this source of endogeneity bias in the estimation process (see the seminal article by [4] in the automobile market context; [6] or [8] in the location choice context; or [10] for a recent survey on endogeneity problems in DCM.

In this paper, we are interested in the allocation process when supply is smaller than demand in some alternatives. Such an allocation has been described as a search mechanism by [5], later on 
applied in the regulated housing market in Holland by [7]. Here we adopt a completely different approach to address the problem of disequilibrium between supply and demand. We assume that the final allocation mechanism should obey some simple explicit rules (or axioms). From these assumptions, we are able to compute the ex post allocation. That is, we only describe the ex ante choice (i.e. the choice which ignores the capacity constraints) and the ex post allocation (i.e. the individual choice once the competition for scarce housing resources has taken place). Therefore, in the proposed approach, we do not specify the complex dynamics which characterizes the adjustment process from ex ante to ex post allocation. Below we describe the organization of the paper.

Following a brief description of the study area and the data used in the empirical work (Section 2), we develop the model specifications and estimation algorithm for markets with constrained availability of at least some alternatives (Section 3). We then present the estimation results and an analysis of the sensitivity of the algorithm (Section 4). The paper concludes (Section 5) with an assessment of the contribution of this research and proposed extensions of it.

\section{The study context}

\subsection{Central Paris and suburbs}

The Ile-de-France is one of the most important economic regions in Europe. It houses 11 million people ( 2 million in Paris) and 5.1 million jobs within a space of $12,000 \mathrm{sq} . \mathrm{km}$. Careful preservation of the historic central city of Paris and its many landmarks has had the effect of making Paris a major tourist mecca for the globe, largely by restricting supply of new housing and office space in the historic core, and protecting historic buildings from alteration or replacement. As a consequence, real estate prices are high in the center, and lower-income housing is concentrated in the several suburbs and new tows, mainly (but not only) in the eastern portion of the region. This is an important motivation for the focus of this paper, since the desirability of central Paris, coupled with strong supply constraints, provides a prime example of the kind of constrained market we wish to analyze. Most of the growth in population and employment are outside the core of the city, fueling rapid suburbanization and growth in travel. The regional express roadway network contains 4,500 lane-km, and despite the traditional rush-hour traffic jams, the average duration of a car trip is still only 19 minutes (EGT, 2001). With a ubiquitous metro system in central Paris and half of households in the city not owning a car, transit ridership is quite high. Half of commuting trips in the region in 2001 were by private cars, with $36 \%$ using public transportation and $14 \%$ using a bicycle or walking, though the transit mode share has declined $6 \%$ over the past twenty years as the region has decentralized. An efficient radial public transport system (RER) and freeways connect Paris to the suburbs.

Most of the households (71\%) are male headed. The "poor households" (that is, the $33 \%$ households in the region with the lowest per capita income, defined as household income divided by the square root of the number of persons in the household) are unevenly distributed in the region: only $26 \%$ of households living in district 78 , to the West of Paris, are poor, whereas this fraction goes up to $41 \%$ in district 93, to the East. These same two districts contain the highest (38\% in district 78$)$ and the lowest (21\% in district 93$)$ concentrations of rich households. 
Single-person households are highly concentrated in Paris city (52\% of households in Paris are single). Between 25 to $30 \%$ percent of households of all the districts have two members. The larger families represent a larger share in rural districts in outer ring. About $25 \%$ percent of households have no working member, and out of these, $28 \%$ percent live in Paris city ${ }^{1}$. Nearly $50 \%$ of the families in outer ring have two or more workers. Foreign households are most concentrated in district 93 (19\%), and are less represented in the outer ring (9\%). About 25\% of households have a young head. They represent a larger share in Paris center and district 92 (31\% and $27 \%)$ and their share is uniform in other districts $(23 \%)$.

\subsection{Geographical units and moves}

The geographic units used in this analysis follow the administrative boundaries used in France. The smallest administrative unit, and the one we use as the basis for the residential location choice model is the commune, of which there are 1300 in the Ile-de-France. These roughly correspond to small cities, in terms of having local administrative control of land use decisions. Note that local administrative control operates at two different levels within Paris: each of the 20 arrondisements has his own mayor, who has less administrative control than the mayor of Paris. However, we will still refer to Paris arrondissements as communes for simplicity. The 1300 communes are grouped into 8 districts (départements).

Table 1 presents the origin and destination rings and districts for the moves observed in 1998, defined as households who changed their location in 1998, with a final destination in Ilede-France. This population of recent movers within the region is the subject of our analysis of the housing market in the Paris region. Most of the moves in 1998 are within the same district, although households who move to Paris come predominantly from outside Ile-de-France, following a classical migration staging pattern observed in many other cities. The most common destination for immigrants from outside Ile-de-France region is Paris, followed by district 92 in the inner ring, which shares many attributes with Paris.

Table (1): The distribution of moves between different rings in 1998 (origin by destination).

\begin{tabular}{|l|l|c|c|c|c|c|}
\hline \multicolumn{7}{|c|}{ Origin district } \\
\hline Current District & & Outside & Paris & Inner Ring & Outer Ring & Total \\
\hline \multirow{2}{*}{ Paris } & Frequency & 77.579 & 67.027 & 18.192 & 18.023 & 180.821 \\
& Percent & $42.9 \%$ & $37.1 \%$ & $10.1 \%$ & $10.0 \%$ & $30.68 \%$ \\
\hline \multirow{2}{*}{ Inner Ring } & Frequency & 61.135 & 22.633 & 103.205 & 20.168 & 207.141 \\
& Percent & $29.5 \%$ & $10.9 \%$ & $49.8 \%$ & $9.7 \%$ & $35.15 \%$ \\
\hline \multirow{2}{*}{ Outer Ring } & Frequency & 49.936 & 9.299 & 23.967 & 118.191 & 201.393 \\
& Percent & $24.8 \%$ & $4.6 \%$ & $11.9 \%$ & $58.7 \%$ & $34.17 \%$ \\
\hline \multirow{2}{*}{ Ile-de-France } & Frequency & 188.650 & 98.959 & 145.364 & 156.382 & 589.355 \\
& Percent & $32.01 \%$ & $16.79 \%$ & $24.66 \%$ & $26.53 \%$ & $100.00 \%$ \\
\hline
\end{tabular}

Source: Census, 1999.

\footnotetext{
${ }^{1}$ This reflects more a larger fraction of retired household members in Paris city than a larger unemployment ratio.
} 


\subsection{Housing Turnover Index (HTI) and supply}

According to the 1999 Census, 8.06\% of the dwellings located in Ile-de-France were vacant in March 1999. While this represents a reasonable estimate of the vacancy rate over a very short period of time, for our analysis, we wish to approximate the availability of housing over a longer period of one year (one year supply). ${ }^{2}$ Unfortunately, we have no information on how long each dwelling was vacant before a household moved in. However, during the period between the time a household moved out and another moved in, the dwelling was vacant and could have been chosen by another household. For this reason, some fraction, denoted by $\lambda$, of the dwellings occupied in March 1999 by a household which moved in 1998 should be included in the one year supply. This fraction $\lambda$ is interpreted as a Housing Turnover Index. If we assume that dwellings are vacant, on average, during half a year $(\lambda=0.5)$, then the one-year supply is made of 409,491 dwellings vacant in March 1999, and half the 589,355 dwellings in which a household moved in 1998 , which represents a total supply of 704,168.5 dwellings in the region. We consider it as an upper bound for the supply, leading to a lower bound for the constraints, while we will also explore smaller values for $\lambda$. More interestingly, the time a dwelling remains vacant is shorter in Paris than in most of the other districts, which means that the HTI $\lambda$ varies across the region. We therefore computed the HTI for each district under a stationarity assumption. ${ }^{3}$ We assume that the ratio of the (cumulated) stock of vacant dwelling to the (cumulated) supply stock is equal to the ratio of the one-year flow of movers to the supply defined above, which can be considered as a one-year supply flow. ${ }^{4}$ Table 2 presents the vacancy rate and HTI $\lambda$ computed by district and shows that, although Paris displays the highest vacancy rate, it also displays the second lowest HTI. The global HTI is 0.39598 for Ile-de-France, which means that dwellings remain vacant on average 4.75 months before they are occupied again.

Table (2): Vacancy rate and Housing Turnover Index.

\begin{tabular}{|l|l|r|l|}
\hline Sub-region & District & Vacancy rate & HTI $\lambda$ \\
\hline Paris & 75 & 0.10325 & 0.36773 \\
\hline Close Suburbs & 92 (West) & 0.08447 & 0.41411 \\
(Inner Ring) & 93 (North) & 0.08461 & 0.28478 \\
& 94 (South) & 0.07479 & 0.38813 \\
\hline & 78 (West) & 0.06188 & 0.49909 \\
Far away suburbs & 91 (South) & 0.06596 & 0.42586 \\
(Outer Ring) & 95 (North) & 0.06286 & 0.45274 \\
& 77 (East) & 0.06557 & 0.44376 \\
\hline
\end{tabular}

\footnotetext{
${ }^{2}$ We observe the households which moved during one year (1998), without information on the exact date they moved. In addition, we know which district they come from, but not which commune they come from. Moreover, we have no information on the number of households who left Ile de France in 1998.

${ }^{3}$ The HTI computed at a lower geographical level would be too sensitive to the randomness of the number of movers during a given year.

${ }^{4}$ Let $M$ and $V$ denote the numbers of movers and vacant units, respectively. The annual supply is $\lambda M+V$, so that the annual supply over demand ratio is $(\lambda M+V) / M$, where $\lambda$ represents the fraction of a year a housing unit remains vacant after a household leaves it. Let $T$ and $S$ denote the total housing stock and the secondary residence units (these dwellings should not be included in the supply, although most of them were not occupied at the time census data were collected), respectively. The aggregate supply over demand ratio is $(T-S) /(T-S-V)$. Equating these two ratios, we find an expression for $\lambda: \lambda=\frac{T-S}{T-S-V}-\frac{V}{M}$.
} 
Source: Author's computations from census data.

\section{Model specification}

In this section, we develop the specification for the model, beginning with the household residential location choice, and elaborating the basic model to address constraints. For clarity of exposition, we begin with a stronger assumption of homogeneous agents (no individual characteristics are considered), and then relax this to accommodate heterogeneous agents. We follow with the description of the iterative solution algorithm, first when the demand is known (parameters are given for the residential location choice model), and then when the demand is unknown. In this latter case, we need to estimate the parameters of the location choice model to check if the capacity constraint in a commune is violated or not, and to separate this constraint effect from the effect of preferences.

\subsection{Household residential location choice: basic model and notation}

We refer to households that make a location choice within a given year as movers. These represent the set of agents producing demand for housing. Supply of housing is considered to be the set of housing units that are available for locating households (see Section 2.3 and footnote 4). The set of alternatives in the housing market are represented by $J=1300$ communes, denoted by $\mathcal{J}$, with $\operatorname{Card}(\mathcal{J})=J$. The demand for alternative $j$ is denoted by $D_{j}$ and the supply (or capacity) of alternative $j$ is denoted by $S_{j}$. Individual decision makers are indexed by $i, i=1 \ldots N$. The conditional utility of individual $i$ selecting alternative $j$ is:

$$
U_{j}^{i}=V_{j}^{i}+\varepsilon_{j}^{i}, i=1 \ldots N, j \in \mathcal{J},
$$

where $V_{j}^{i}$ represents the systematic component of the utility and where $\varepsilon_{j}^{i}$ are i.i.d. random variables, with a double exponential distribution with a variance equal to $\mu^{2} \pi^{2} / 6$, where $\mu$ is a positive scale parameter. In this case, the probability $\mathbb{P}_{j}^{i}$ that individual $i$ prefers alternative (commune) $j$ is given by the multinomial logit formula (See [2] for details):

$$
\mathbb{P}_{j}^{i}=\frac{\exp \left(V_{j}^{i} / \mu\right)}{\sum_{j^{\prime} \in \mathcal{J}} \exp \left(V_{j^{\prime}}^{i} / \mu\right)}, i=1 \ldots N, j \in \mathcal{J} .
$$

In the homogeneous case where we treat agents as though they were identical, systematic utilities and choice probabilities do not vary between individuals, so they are denoted by $V_{j}$ and $\mathbb{P}_{j}=$ $\frac{\exp \left(V_{j} / \mu\right)}{\sum_{j^{\prime} \in \mathcal{J}} \exp \left(V_{j^{\prime}} / \mu\right)}$, respectively. The expected demand, $D_{j}$, for alternative $j$ is:

$$
D_{j}=\left\{\begin{array}{l}
\sum_{i=1}^{N} \mathbb{P}_{j}^{i} \text { in the heterogeneous case } \\
N \mathbb{P}_{j} \text { in the homogeneous case }
\end{array}, j \in \mathcal{J} .\right.
$$




\subsection{Introducing capacity constraints}

The analysis of the residential choices in Ile-de-France (based on preliminary demand estimates) shows that many alternatives (about half, depending on the specification) have greater demand than supply. We consider below the situation where demand $D_{j}$ strictly exceeds supply $S_{j}$ for at least one alternative $j$. In that case, we say that the system is constrained. We denote $D_{j}-S_{j}$ as the excess demand, which is positive for at least one alternative when the system is constrained. When the system is constrained, the choices based only on preferences, or ex ante choices, differ from the actual allocation consistent with preferences and with capacity constraints, or ex post allocation, because the constraints are binding and some households are forced to take an alternative which was not their first preference. Note that even when only one alternative is constrained, ex post demand is a priori different from ex ante demand, for all alternatives. We refer to the demand after constraints are imposed as the ex post demand. It is in this sense that we will refer to equilibrium, or market clearing. The time frame for the analysis is considered short-term, with supply assumed to be constant.

In order to guarantee that there exists at least one feasible allocation, we also assume that constraints are not globally too severe. That is, we impose a feasibility condition that aggregate supply is sufficient to accommodate aggregate demand, resulting in a vacancy rate that is strictly positive:

$$
N=\sum_{j \in \mathcal{J}} D_{j}<\sum_{j \in \mathcal{J}} S_{j}
$$

When the system is constrained, the (ex post) probability that individual $i$ is allocated to alternative $j$ is denoted by $\tilde{\mathbb{P}}_{j}$ in the homogeneous case (or by $\tilde{\mathbb{P}}_{j}^{i}$ in the heterogeneous case). The ex ante demand $D_{j}$ defined in (3) corresponds to ex ante choices, whereas the observed demand (or ex post demand), denoted by $\tilde{D}_{j}$, corresponds to the ex post allocation:

$$
\tilde{D}_{j}=\left\{\begin{array}{l}
\sum_{N \tilde{\mathbb{P}}_{j}=1}^{N} \tilde{\mathbb{P}}_{j}^{i} \text { in the homogeneous case } \\
\sum^{2} \text { the herogeneous case }
\end{array} .\right.
$$

Note that, when the system is not constrained, then $\tilde{\mathbb{P}}_{j}^{i}=\mathbb{P}_{j}^{i}, j \in \mathcal{J}$, for all alternatives and all individuals, and the observed demand is equal to the ex ante demand for all alternatives. When the system is constrained, $\tilde{D}_{j}$ is bounded by $S_{j}(j \in \mathcal{J})$ and the constraint is binding for at least one alternative. For a given alternative $j$, two situations may arise:

1. Either alternative $j$ is unconstrained ex ante: $D_{j}<S_{j}$. In this case, alternative $j$ is unconstrained ex post if $\tilde{D}_{j}<S_{j}$, while alternative $j$ is constrained ex post if $\tilde{D}_{j}=S_{j}$.

2. Or alternative $j$ is constrained ex ante: $D_{j} \geq S_{j}$. In this case, it can be shown, under simple regularity conditions, that $\tilde{D}_{j}=S_{j}$, i.e. alternative $j$ is also constrained ex post.

We define the following sets of unconstrained alternatives: $\overline{\mathcal{C}}=\left\{j \in \mathcal{J} \mid \tilde{D}_{j}<S_{j}\right\}$ denotes the alternatives unconstrained ex post, while $\overline{\mathcal{C}}(0)=\left\{j \in \mathcal{J} \mid D_{j}<S_{j}\right\}$ denotes the alternatives unconstrained ex ante.

Similarly, we define the following sets of constrained alternatives: $\mathcal{C}=\left\{j \in \mathcal{J} \mid \tilde{D}_{j}=S_{j}\right\}$ denotes the alternatives constrained ex post, while $\mathcal{C}(0)=\left\{j \in \mathcal{J} \mid D_{j} \geq S_{j}\right\}$ denotes the set of alternatives constrained ex ante.

These four sets determine two partitions of $\mathcal{J}($ since $\mathcal{C} \cap \overline{\mathcal{C}}=\mathcal{C}(0) \cap \overline{\mathcal{C}}(0)=\varnothing$ and $\mathcal{C} \cup \overline{\mathcal{C}}=\mathcal{C}(0) \cup$ $\overline{\mathcal{C}}(0)=\mathcal{J})$. When the system is constrained, the actual allocation is the result of a complex 
mechanism which depends on the assumptions (priority rules) introduced below. Such assumptions characterize ex post allocation.

Constraints have two consequences: first, if alternative $j$ is constrained ex ante $(j \in \mathcal{C}(0))$, a fraction of the individuals who would select alternative $j$ without capacity constraints $\left(D_{j}\right)$ must instead choose a less desirable alternative $k$; second, due to the first consequence, the excess demand generated in alternatives constrained ex ante is reallocated to alternatives which were not constrained ex ante. Therefore, the observed demand is larger than (or equal to) the ex ante demand in all alternatives unconstrained ex ante. Some of the alternatives which are not constrained ex ante may be constrained ex post, due to the reallocation of excess demand.

We now introduce a first assumption: free allocation, which means that if an individual prefers an alternative $j$, which is unconstrained ex post, he can be sure to be allocated to it.

Assumption 1 (Free allocation) Let $j \in \overline{\mathcal{C}}$. Then

$$
\mathbb{P}(\{i \text { allocated to } j \mid i \text { prefers } j\})=1, \forall i=1 \ldots N \text {. }
$$

However, note that an individual may be denied access to his preferred choice $j$ if it is constrained ex post, and may be forced to choose another alternative (that may be constrained or unconstrained ex ante). Assumption 1 implies that the IIA property (specific to the MNL model) is valid for the unconstrained alternative, that is, for two alternatives unconstrained ex post, the ratio of (ex post) allocation probabilities is equal to the ratio of (ex ante) choice probabilities. Assumption 1 implies the IIA property in the following sense:

Lemma 1 (Alternative unconstrained ex post) If Assumption 1 holds and if alternative $j$ and $k$ are unconstrained ex post, then the allocation probabilities satisfy the IIA property in the sense that:

$$
\frac{\tilde{\mathbb{P}}_{j}^{i}}{\tilde{\mathbb{P}}_{k}^{i}}=\frac{\mathbb{P}_{j}^{i}}{\mathbb{P}_{k}^{i}}, \forall i=1 \ldots N, \forall j, k \in \overline{\mathcal{C}} .
$$

Moreover:

$$
\tilde{\mathbb{P}}_{j}^{i} \geq \mathbb{P}_{j}^{i} \text { and } \tilde{D}_{j} \geq D_{j}, \forall i=1 \ldots N, \forall j \in \overline{\mathcal{C}} .
$$

The equality (6) states that the allocation probabilities satisfy the IIA property for unconstrained alternatives. The inequalities (7) state that the probability that individual $i$ is allocated to $j$ is larger than the probability that he prefers $j$ and the observed demand addressed to $j$ is larger than the ex ante demand if $j$ is unconstrained. An immediate consequence of Assumption 1 and Lemma 1 is that if alternative $j$ is constrained ex ante, then it is also constrained ex post; if alternative $j$ is unconstrained ex post, then it is also unconstrained ex ante.(in formal terms, $\mathcal{C}(0) \subset \mathcal{C}$ and $\overline{\mathcal{C}} \subset \overline{\mathcal{C}}(0))$.

Lemma (1) states that the individual ratio $\tilde{\mathbb{P}}_{j}^{i} / \mathbb{P}_{j}^{i}$ of the actual allocation probability to the choice probability is the same across all unconstrained alternatives, since equality (6) can be rewritten as:

$$
\frac{\tilde{\mathbb{P}}_{j}^{i}}{\mathbb{P}_{j}^{i}}=\frac{\tilde{\mathbb{P}}_{k}^{i}}{\mathbb{P}_{k}^{i}} \stackrel{\text { def }}{=} \Omega^{i}, \forall i=1 \ldots N, \forall j, k \in \overline{\mathcal{C}}
$$


where the common value of this ratio is denoted by $\Omega^{i}$. We interpret $\Omega^{i}$ as the individual allocation ratio for individual $i$. When $\Omega^{i}=1.1$, for example, this implies that, for any unconstrained alternative $j$, the probability that individual $i$ is allocated to alternative $j$ is $10 \%$ higher than the probability that $i$ prefers $j$. This also means that, if individual $i$ is allocated to an alternative $j$ unconstrained ex post, the probability that $j$ is not his/her preferred alternative is $9.09 \%$ $\left(\frac{\tilde{\mathbb{P}}_{j}^{i}-\mathbb{P}_{j}^{i}}{\widetilde{\mathbb{P}}_{j}^{i}}=\frac{\Omega^{i}-1}{\Omega^{i}}\right)$. The ratio $\frac{\Omega^{i}-1}{\Omega^{i}}$, which does not depend on $j$, therefore corresponds to the probability that $i$ is not allocated to his/her preferred alternative, given that he/she is allocated to an alternative unconstrained ex post. Note that (7) implies $\Omega^{i}>1$, so $\frac{\Omega^{i}-1}{\Omega^{i}}>0$.

The computation of $\Omega^{i}$ cannot be performed before the allocation rules are defined for the ex post constrained alternatives. We now introduce a second assumption concerning the allocation in alternatives which are constrained ex post. We assume that if an individual $i$ has a stronger preference (ex ante) for the alternative $j$ constrained ex post than another individual $i^{\prime}$, in the sense that the choice probability for $i$ is larger than the choice probability for $i^{\prime}$, individual $i$ will also have proportionally more chances than individual $i^{\prime}$ to be allocated ex post to alternative $j$ in the following sense:

Assumption 2 (No priority rule) If $j \in \mathcal{C}$, the individual allocation ratio of alternative $j$, constrained ex post is the same for all individuals:

$$
\frac{\tilde{\mathbb{P}}_{j}^{i}}{\mathbb{P}_{j}^{i}}=\frac{\tilde{\mathbb{P}}_{j}^{i^{\prime}}}{\mathbb{P}_{j}^{i^{\prime}}} \stackrel{\text { def }}{=} \Phi_{j}, \forall i, i^{\prime}=1 \ldots N, \forall j \in \mathcal{C} .
$$

Assumption 2 states that the individual allocation ratio for alternative $j$, denoted by $\Phi_{j}$, is the same for each individual $(i=1 \ldots N)$. Assumption 2 can therefore be interpreted as a fairness criterion.

This assumption suffices to determine the queue discipline for the alternatives which are constrained ex post. Indeed, it is straightforward to show that the common value (across individuals) of the allocation ratio $\tilde{\mathbb{P}}_{j}^{i} / \mathbb{P}_{j}^{i}$ is equal to the relative supply (measured by $S_{j} / D_{j}$ ):

Lemma 2 (Alternative constrained ex post) If Assumption 2 holds and alternative $j$ is constrained ex post, the common value of the individual allocation ratio is equal to the relative supply, i.e.:

$$
\Phi_{j}=\frac{S_{j}}{D_{j}}<1, \forall j \in \mathcal{C}
$$

Assumptions 1 and 2 hold, throughout the rest of the paper. They are essentially sufficient to solve for an equilibrium. It is necessary, also, to eliminate extreme preferences (which are hardly observed in our data), which corresponds to a kind of "no preference reversal" assumption. ${ }^{5}$

We provide below the solution for the homogeneous case and sketch the solution for the heterogenous case.

\footnotetext{
${ }^{5}$ We need to eliminate extreme preferences. With that respect, one supplementary assumption is the "no preference reversal" : no individual in the population has a strong preference for alternatives, which are constrained ex post but not ex ante. More specifically, we assume that $\sum_{j \in \mathcal{C} \cap \overline{\mathcal{C}}(0)} \mathbb{P}_{j}^{i}$ is not too large.
} 


\subsection{Equilibrium solution with capacity constraints}

The solution requires the computation of two unknown pieces of information: (1) which alternatives are constrained ex post and (2) the value of $\Omega^{i}, i=1 \ldots N$. We assume for the moment that we know which alternatives are constrained ex post and will return to the computation of this list subsequently.

Recall that, for an unconstrained alternative $j$, the individual allocation ratio, $\tilde{\mathbb{P}}_{j}^{i} / \mathbb{P}_{j}^{i}=\Omega^{i}$, is independent of the alternative. For any alternative $j$ unconstrained ex post, the individual allocation ratio $\tilde{\mathbb{P}}_{j}^{i} / \mathbb{P}_{j}^{i}$ is larger than 1 since some individuals are reallocated from their preferred constrained alternative to alternative $j$, whereas, according to Assumption 1, all individuals who prefer $j$ are allocated to $j$. The value of the individual allocation ratio in unconstrained alternatives is given by:

Lemma 3 (Individual allocation ratio) Consider an individual $i$. The individual allocation ratio is the same for each alternative unconstrained ex post and is given by:

$$
\frac{\tilde{\mathbb{P}}_{j}^{i}}{\mathbb{P}_{j}^{i}}=\Omega^{i}=\frac{1-\sum_{j \in \mathcal{C}} \frac{S_{j}}{D_{j}} \mathbb{P}_{j}^{i}}{\sum_{k \in \overline{\mathcal{C}}} \mathbb{P}_{k}^{i}}>1, \forall j \in \overline{\mathcal{C}} .
$$

Lemma 3 shows that the individual allocation ratio $\Omega^{i}=\tilde{\mathbb{P}}_{j}^{i} / \mathbb{P}_{j}^{i}, \forall j \in \overline{\mathcal{C}}$ is uniquely determined once the set $\mathcal{C}$ of alternatives constrained ex post is known. Note that $\Omega^{i}=\frac{1-\sum_{j \in \mathcal{C}} \frac{S_{j}}{D_{j}} \mathbb{P}_{j}^{i}}{1-\sum_{j \in \mathcal{C}} \mathbb{P}_{j}^{i}}$, which is larger than 1 since $\frac{S_{j}}{D_{j}}<1, \forall j \in \mathcal{C}(0)$ and $\sum_{j \in \mathcal{C} \cap \overline{\mathcal{C}}(0)} \mathbb{P}_{j}^{i}$ is not too large (see footnote $5)$, so that the negative difference $\sum_{j \in \mathcal{C} \cap \overline{\mathcal{C}}(0)}\left(\mathbb{P}_{j}^{i}-\frac{S_{j}}{D_{j}} \mathbb{P}_{j}^{i}\right)$ cannot offset the positive difference $\sum_{j \in \mathcal{C}(0)}\left(\mathbb{P}_{j}^{i}-\frac{S_{j}}{D_{j}} \mathbb{P}_{j}^{i}\right)$.

The ex ante demand is given by (3), while the ex post demand is defined by: $\tilde{D}_{j}=\sum_{i=1}^{N} \tilde{\mathbb{P}}_{j}^{i}=$ $\sum_{i=1}^{N} \Omega^{i} \mathbb{P}_{j}^{i}$ (see equation (5)). The aggregate allocation ratio, $\bar{\Omega}_{j}$, is defined as a weighted average of individual allocation ratios $\Omega^{i}$ :

$$
\bar{\Omega}_{j} \stackrel{\text { def }}{=} \frac{\sum_{i=1}^{N} \Omega^{i} \mathbb{P}_{j}^{i}}{\sum_{i=1}^{N} \mathbb{P}_{j}^{i}}>1, \forall j \in \mathcal{J} .
$$

Note that $\bar{\Omega}_{j}>1$ if $j \in \overline{\mathcal{C}}$ (see Lemma 3 ). In this case, we have (using (3)):

$$
\tilde{D}_{j}=\bar{\Omega}_{j} D_{j} \text { and } \tilde{\mathbb{P}}_{j}^{i}=\Omega^{i} \mathbb{P}_{j}^{i}, \forall j \in \overline{\mathcal{C}} .
$$

For example, $\bar{\Omega}_{j}=1.1$ means that observed demand $\tilde{D}_{j}$ is $10 \%$ higher than the expected demand $D_{j}$. This also means that $9.09 \%$ of the individuals allocated to alternative $j$ prefer another alternative (see also discussion of Equation (8)). Collecting the previous results, we have: 
Lemma 4 (Aggregate allocation ratio) Consider an alternative unconstrained ex post. The alternative-specific aggregate allocation ratio is :

$$
\bar{\Omega}_{j}=\frac{\sum_{i=1}^{N} \Omega^{i} \mathbb{P}_{j}^{i}}{\sum_{i=1}^{N} \mathbb{P}_{j}^{i}}=\frac{\tilde{D}_{j}}{D_{j}}, j \in \overline{\mathcal{C}},
$$

where $\Omega^{i}$ are given by Lemma 3.

Recall that the value of the individual allocation ratio $\Omega^{i}$ can be computed once the set $\mathcal{C}$ is known (see Lemma 3). Conversely, assume that the ratios $\Omega^{i}$ (and therefore $\bar{\Omega}_{j}$ ) are known. It can be shown that an alternative $j$ is constrained ex post if and only if $\bar{\Omega}_{j} D_{j}=\sum_{i=1}^{N} \Omega^{i} \mathbb{P}_{j}^{i}$ is larger than (or equal to) $S_{j}$. Therefore, we have:

$$
j \in \overline{\mathcal{C}} \Leftrightarrow \bar{\Omega}_{j} \geq \frac{S_{j}}{D_{j}} .
$$

Note that, if alternative $j$ is constrained ex ante, then $S_{j} / D_{j}<1$, and the condition (10) is always satisfied (ex ante constraint implies ex post constraint). If alternative $j$ is constrained ex post but not ex ante, then $S_{j} / D_{j} \geq 1$, but $\bar{\Omega}_{j}$ is even larger. In both cases $(j \in \mathcal{C}(0)$ and $j \in \mathcal{C} \cap \overline{\mathcal{C}}(0))$, we have:

$$
\tilde{D}_{j}=S_{j} \text { and } \tilde{\mathbb{P}}_{j}^{i}=\frac{S_{j}}{D_{j}} \mathbb{P}_{j}^{i}, j \in \mathcal{C} .
$$

The proofs of existence and uniqueness of a global solution $\left(\Omega^{i}, \mathcal{C}\right)$ remain an open issue (although numerical simulation suggest that existence and uniqueness are guaranteed).

Below, we provide a method for computing the allocation equilibrium, either when the demand is known and the parameters are given, or when it is unknown and the parameters need to be estimated in tandem with the iterative procedure.

\subsection{Computational method}

\subsubsection{Allocation Probabilities}

The allocation probabilities are given by different expressions according to whether the alternatives are constrained or not ex post. The allocation probabilities can still be written as a multinomial logit model, but with an additional term, or "correction factor", $\ln \left(\pi_{j}^{i}\right)$, which expresses the allocation ratio.

Theorem 1 (Allocation probabilities) If Assumptions 1 and 2 hold, the allocation probabilities are given by the adjusted $M N L$ formula:

$$
\begin{aligned}
& \tilde{\mathbb{P}}_{j}^{i}=\frac{\exp \left(\tilde{V}_{j}^{i} / \mu\right)}{\sum_{k \in \mathcal{J}} \exp \left(\tilde{V}_{k}^{i} / \mu\right)}, \text { with } \\
& \tilde{V}_{j}^{i}= V_{j}^{i}+\mu \ln \left(\pi_{j}^{i}\right), \text { with } \\
& \pi_{j}^{i}=\left\{\begin{array}{l}
\frac{S_{j}}{D_{j}} \text { if } j \in \mathcal{C} \\
\Omega^{i} \text { if } j \in \overline{\mathcal{C}} .
\end{array}\right.
\end{aligned}
$$


Proof. The denominator in (11a) is

$$
\begin{gathered}
\sum_{k \in \mathcal{J}} \exp \left(\tilde{V}_{k}^{i} / \mu\right)=\sum_{k \in \mathcal{C}} \frac{S_{k}}{D_{k}} \exp \left(V_{k}^{i} / \mu\right)+\Omega^{i} \times \sum_{k \in \overline{\mathcal{C}}} \exp \left(V_{k}^{i} / \mu\right) \\
=\sum_{j \in \mathcal{J}} \exp \left(V_{j}^{i} / \mu\right) \times\left[\sum_{k \in \mathcal{C}} \frac{S_{k}}{D_{k}} \frac{\exp \left(V_{k}^{i} / \mu\right)}{\sum_{j \in \mathcal{J}} \exp \left(V_{j}^{i} / \mu\right)}+\Omega^{i} \sum_{k \in \overline{\mathcal{C}}} \frac{\exp \left(V_{k}^{i} / \mu\right)}{\sum_{j \in \mathcal{J}} \exp \left(V_{j}^{i} / \mu\right)}\right] \\
=\sum_{j \in \mathcal{J}} \exp \left(V_{j}^{i} / \mu\right) \times\left[\sum_{k \in \mathcal{C}} \frac{S_{k}}{D_{k}} \mathbb{P}_{k}^{i}+\Omega^{i} \sum_{k \in \overline{\mathcal{C}}} \mathbb{P}_{k}^{i}\right] \\
=\sum_{j \in \mathcal{J}} \exp \left(V_{j}^{i} / \mu\right) \times \sum_{j \in \mathcal{J}} \tilde{\mathbb{P}}_{j}^{i}=\sum_{j \in \mathcal{J}} \exp \left(V_{j}^{i} / \mu\right) .
\end{gathered}
$$

Therefore:

$$
\frac{\exp \left(\tilde{V}_{j}^{i} / \mu\right)}{\sum_{k \in \mathcal{J}} \exp \left(\tilde{V}_{k}^{i} / \mu\right)}=\pi_{j}^{i} \times \frac{\exp \left(V_{j}^{i} / \mu\right)}{\sum_{j \in \mathcal{J}} \exp \left(V_{j}^{i} / \mu\right)}=\pi_{j}^{i} \mathbb{P}_{j}^{i}=\tilde{\mathbb{P}}_{j}^{i}
$$

The reader should still keep in mind that the difficult part of this approach (and of any approach dealing with constraints) is the determination of the set of alternatives which are constrained ex post.

We first describe the iterative procedure to find the allocation probabilities and the ex post constrained alternatives, when the demand is known, as would be the case once the model is estimated and is being used to make predictions. This simplifies the initial exposition. Next we treat the case when demand is unknown and has to be estimated.

\subsubsection{Algorithm K: the solution algorithm with known demand}

We now turn to the development of the solution algorithm for the model, beginning with the simpler circumstance in which the utility parameters are assumed to be known a priori. We refer to this case as known demand, and its solution algorithm as Algorithm K. The formulas are given by Lemma 3 for the individual allocation ratio $\Omega^{i}$, by Lemma 4 for the aggregate allocation ratio $\bar{\Omega}_{j}$ and by condition (10) for the set $\mathcal{C}$ of alternatives $j$ constrained ex post. The algorithm allows computation of $\Omega^{i}, \bar{\Omega}_{j}$ and $\mathcal{C}$ with less than $J$ iterations. The details of the Algorithm K are included in the Appendix. In heuristic terms, the algorithm iteratively estimates the constraints and the individual and aggregate allocation ratios until they converge.

\subsubsection{Algorithm U: the solution algorithm with unknown demand}

When the utility parameters are unknown, we need to jointly estimate the parameters of the model and to determine the allocated demand, given a set of parameters. We accomplish this by creating a new Algorithm U that embeds Algorithm K as an inner loop, and adds an outer 
loop that generates estimates of the utility parameters and the resulting demand. The approach is to iterate between a utility parameter estimation phase, which incorporates the correcting factor $\ln \left(\pi_{j}^{i}\right)$, computed during the previous iteration and an inner loop based on Algorithm $\mathrm{K}$, which in turn relies on the previous iteration estimated demand. The outer loop (demand estimation) is indexed by $l^{\prime}$ and the inner loop (constraints) is indexed by $l$.

We require additional notations: $\hat{D}_{j}\left(l^{\prime}\right), \hat{\mathbb{P}}_{j}^{i}\left(l^{\prime}\right)$ and $\ln \left[\pi_{j}^{i}\left(l^{\prime}\right)\right]$ represent the estimation of the demand, the choice probabilities and the correction factor at iteration $l^{\prime}$. Moreover, $\mathcal{C}\left(l^{\prime}, l\right)$ denotes the set of alternatives constrained ex post at iteration $\left(l^{\prime}, l\right)$, where $l^{\prime}$ corresponds to the demand estimation and $l$ corresponds to the constraints. We define $\Omega^{i}\left(l^{\prime}, l\right)$ and $\bar{\Omega}_{j}\left(l^{\prime}, l\right)$ in a similar way. We provide the structure of the solution algorithm when unknown demand is estimated in the Appendix, labelled Algorithm U. In the next section, we use Algorithm $\mathrm{U}$ to estimate the residential location demand model when the system is constrained. In the simulation results reported (and not reported), Algorithm $U$ always converged to a unique solution.

\section{Empirical results}

\subsection{Data}

The household data come from the 1999 census, for which we were able to access household-level data for the entire population, allowing us to test the sensitivity of the estimation to using a range of sampling rates from the population, a range of numbers of alternatives and various specifications for demand equation and supply computation (value of the Housing Turnover Index). The analysis focuses on "recent movers": households who settled or moved to the region recently, that is during year 1998. Among the 4,510,369 households living in the study area in March 1999, 589,355 moved into or within the region of interest during year 1998.

The housing price data used in the model come from the "base de données des Notaires" (notaries' database) and contains the average price of single-family dwellings sold in the commune in 1998. We do not observe prices for single dwellings, but only the number of dwellings sold and the average price in each commune. Table 3 presents statistics computed with one observation (average price) per commune, weighted by the number of dwellings sold in that commune during year 1998. Differences in average housing prices by district are large: prices are higher in Paris, intermediate in the close suburbs and decline in the more distant suburbs, which we refer to as the inner ring and the outer ring, respectively. In addition, prices are higher in the western part of the area than in the eastern part, consistent with the spatial patterns of social stratification. Note that the heterogeneity of prices is probably underestimated when using such average prices computed at the commune level, because they do not correct for the characteristics of the dwellings, which are typically large houses in the West suburbs and small flats inside Paris. This suggests that the differences between Paris and the suburbs reported in Table 3 are smaller than differences in price per square meter, and this may explain why the largest average price is observed in district 92, and is nearly twice the largest average price inside Paris. See [6] for additional details on the data, and for price endogeneity tests. 
Table (3): Prices by district (in year 1998).

\begin{tabular}{|l|l|r|r|r|r|}
\hline Sub-region & District & Average & $\begin{array}{l}\text { Standard } \\
\text { Deviation }\end{array}$ & Minimum & Maximum \\
\hline Paris & 75 & 294,500 & 165,241 & 83,939 & 694,375 \\
\hline Close Suburbs & 92 (West) & 247,556 & 205,038 & 66,966 & $1,198,950$ \\
(Inner Ring) & 93 (North) & 115,709 & 49,055 & 47,876 & 259,163 \\
& 94 (South) & 144,098 & 74,603 & 53,356 & 373,499 \\
\hline & 78 (West) & 135,122 & 65,714 & 38,112 & 373,815 \\
Far away suburbs & 91 (South) & 114,826 & 46,740 & 24,719 & 332,338 \\
(Outer Ring) & 95 (North) & 104,375 & 41,670 & 25,154 & 241,692 \\
& 77 (East) & 91,539 & 37,220 & 18,028 & 253,827 \\
\hline
\end{tabular}

Source: Author's computations from notaries' database.

The attributes of the alternatives have been computed from different sources, mainly drawing on data from the IAURIF metropolitan planning agency. Several variables in the household location choice model warrant a brief description. "Same district as before" is a dummy variable coded 1 when a household lived in the same District as the commune represented by an alternative. Paris is a dummy variable coded 1 if the commune is in Paris. The travel times for TC correspond to transit mode, whereas those by VP correspond to private vehicle (auto) mode. "Distance to highway" is a linear distance of the centroid of the commune to the nearest highway. Social interaction terms are included as the percentage of households in a commune having a certain characteristic, interacted with a dummy variable for whether the locating household possesses a certain characteristic. Remaining variables used in the household location choice model are self-explanatory characteristics of communes.

\subsection{Location choice without capacity constraints}

The variables used in the initial model specification, assuming no constraints on availability of alternatives, are shown in Table 4. Note that variables describing alternative locations that are expected to have a similar influence on the utility of all types of households are entered with no interactions, whereas all household characteristics included in the specification are entered only through interactions with characteristics of alternatives. This is due to the fact that household characteristics do not vary across the alternatives, whereas characteristics of the alternatives do. Including a household characteristic individually would not allow the maximum likelihood estimation algorithm to converge, since there is no way to estimate a parameter on a variable that is constant across all alternatives. We have selected specific household characteristics to interact with a subset of alternative characteristics, in order to capture the most important sources of systematic heterogeneity in preferences across market segments in the population. In particular, household size, income, number or workers, and whether the household is foreign-born are expected to produce potentially significant differences in the utility of the social composition of the commune in terms of social sorting, or residential segregation.

Given the large size of the databases, there is a trade-off between the number of households, the number of alternatives and the number of explanatory variables which can be used for estimating the model. Table 4 contains the results of the residential location choice model estimation, estimated on a $0.5 \%$ sample of households (that is 2,946 households) with all the 
alternatives, and assuming that the system is not constrained (iteration $l^{\prime}=0$ in algorithm $\mathrm{U}$ ). With a pseudo- $\mathrm{R}^{2}$ (McFadden's likelihood ratio index) of $30.6 \%$, this model has a rather good explanatory power at the individual level. Later on, we will explore the validity of this model at the aggregate level (see Section 4.4.4). We find a very significant effect of the "same district as before move" variable, confirming (as expected) a strong preference of households to move in the same district or neighborhood in which they lived before the move. ${ }^{6}$ The "Paris" coefficient is not significant, suggesting that the relative attractiveness of Paris is captured in local amenities and other explanatory variables.

As expected, housing price has a negative effect on location preference for a commune. We tested for the endogeneity of prices in the location choice model introducing the residual of a hedonic price equation (results not reported here). Adding the residuals of the price equation as an explanatory variable, the estimated coefficients changes trivially and the coefficient of this new variable is not at all significant. This result confirms that housing price is not endogenous (in the econometric sense) with regard to the location choice model. In other words, the variables used in these two models fully explain the correlation between prices and location choice. Price effect increases with the age of the household head and decreases as the household income increases. The older heads of households are more sensitive to price and the richer households are less sensitive to it. Since price is entered using three variables to capture average effects as well as interactions with age and income, the combined effects are complex. We note that the average price effect as well as the age and income interactions, all have expected signs. However, for a small subset of the population, namely very young and very rich households, the net price effect from the interaction of these three coefficients would be predicted by this model to show a slight positive preference for higher prices in communes where the neighboring households are in the same socioeconomic category and which have more amenities. The relative sensitivity to price is as expected, though the potential for a small positive preference for higher prices for this specific subpopulation and sample of locations is likely to be due to some amenities that are not accounted for in the model, rather than an actual preference for a higher price, ceteris paribus. In that case, the price effect would become negative for all households if amenities were totally controlled for (see, e.g., [8]).

In this specification, increase of the average travel time by public transit decreases equally the preference of households headed by a man or by a woman. On the opposite, location is not sensitive, ceteris paribus, to the average travel time by private car, or to the distance to highway. These last results are probably explained by the large correlation between "TC" (public transit), "VP" (private car) and "Distance to highway" variables.

In this specification, the demand is not sensitive either to the number of subway (metro) or railway stations. This may also be explained by a large correlation with other explanatory variables such as the "Paris" dummy.

Table (4): Residential location choice, all alternatives, no constraints (0.5\% sample)

\footnotetext{
${ }^{6}$ Testing the effect of the distance from last residence would be interesting, but it was not possible to perform this test with our available data, which did not contain information on previous residence location more detailed than the district.
} 


\begin{tabular}{|c|c|c|c|}
\hline Variable & Coefficient & Std error & t-stat. \\
\hline Same district as before move & 2.585 & 0.047 & 54.80 \\
\hline Paris & 0.047 & 0.128 & 0.364 \\
\hline Log(Price) & -7.108 & 1.445 & -4.920 \\
\hline Log(Price) ${ }^{*}($ Age- 20$) / 10$ & -0.220 & 0.0473 & -4.649 \\
\hline $\log (\text { Price })^{*} \log ($ Income $)$ & 0.746 & 0.146 & 5.110 \\
\hline Number Railway stations & 0.0053 & 0.0151 & 0.352 \\
\hline Number Subway stations & -0.0011 & 0.0070 & -0.156 \\
\hline Average travel time from $\mathrm{j}$, commuting (TC) [hour] & -0.635 & 0.157 & -4.050 \\
\hline TC*(Dummy female) [hour] & -0.066 & 0.231 & -0.287 \\
\hline Average travel time from $\mathrm{j}$, by private car (VP) [hour] & 0.219 & 0.265 & 0.827 \\
\hline Distance to highway $\left[10^{3} \mathrm{~km}\right]$ & -3.238 & 3.441 & -0.941 \\
\hline$\%$ households with 1 member $* 1$ member in $\mathrm{h}$ & 3.769 & 0.452 & 8.336 \\
\hline$\%$ households with 2 members ${ }^{*} 2$ members in $\mathrm{h}$ & -0.591 & 1.678 & -0.352 \\
\hline$\%$ households with $3+$ members $* 3+$ member in $\mathrm{h}$ & 2.523 & 0.455 & 5.549 \\
\hline$\%$ hh with no working memb. ${ }^{*}$ no working memb. in $\mathrm{h}$ & 6.489 & 1.283 & 5.059 \\
\hline$\%$ hh with 1 working memb. ${ }^{*} 1$ working memb. in $\mathrm{h}$ & 0.855 & 0.789 & 1.085 \\
\hline$\%$ hh with $2+$ working memb. ${ }^{*} 2+$ working memb. in $\mathrm{h}$ & -0.890 & 0.556 & -1.601 \\
\hline$\%$ poor households ${ }^{*} \mathrm{~h}$ poor & 1.459 & 0.664 & 2.197 \\
\hline$\%$ households with a foreign head ${ }^{*}$ foreign head in $\mathrm{h}$ & 5.125 & 0.911 & 5.628 \\
\hline$\%$ households with a foreign head ${ }^{*}$ French head in $\mathrm{h}$ & -3.575 & 0.556 & -6.432 \\
\hline Employment $[1,000]$ & 0.263 & 1.292 & 0.204 \\
\hline Density (Population/Surface) [1000 persons $/ \mathrm{km}]$ & -6.625 & 4.360 & -1.52 \\
\hline Log(Population) & 1.011 & 0.029 & 35.00 \\
\hline \% change in population, 1990 to 1999 & 0.185 & 0.097 & 1.898 \\
\hline
\end{tabular}

The estimated coefficients corresponding to the socioeconomic structure of the commune show a general preference of the households to live with neighbors of the same social category. This preference is very strong for singles, for large families, for households without workers, or with a foreign head. Households of French origin tend to avoid locations in which there are higher concentrations of foreign households. The coefficient for the total number of jobs is insignificant in this specification. Households prefer less dense communes, and the communes that have absorbed more population during the 1990-99 period attract still more households. Note that the very large and significant coefficient of "Log(Population)" should not be interpreted as a preference for more populated communes but rather as a proxy for a series of commune dummies allowing a better fit for aggregate demand.

\subsection{Sensitivity analysis}

In order to determine how robust these results are with respect to the sampling procedures we are using, we present below the results of sensitivity analyses to test for the effects different sizes of sampled alternatives. Estimates were also performed to test the effect of different sampling rates for households, with a benchmark $0.5 \%$ of households and 100 alternatives. The results, not reported here in order to save place, are available on demand. They confirm that the variances 
decrease proportionally to sample size, and that estimated coefficients do not vary significantly with households sampling rate.

Table 5 presents the results when 100, 200, 500 and all alternatives are considered for each household. When only a fraction of alternatives is considered, they are selected randomly using an importance sampling strategy (at the commune level). More specifically, the probability of inclusion is proportional to the number of dwellings in the commune; each alternative is selected independently from the other alternatives considered for the same household and for the other households, and repetitions are allowed. This is equivalent to a uniform sampling of dwellings. ${ }^{7}$ The last column in Table 5 corresponds to the coefficients reported in Table 4 . In order to save space, the significance of the coefficients is indicated using signs $(*, \dagger$ and $\ddagger)$.

Table (5): Impact of the number of alternatives on residential location choice ( $0.5 \%$ sample)

\begin{tabular}{|c|c|c|c|c|}
\hline Variable \Household Sampling Rate & 100 & 200 & 500 & 1300 \\
\hline Same district as before move & $2.578 \ddagger$ & $2.583 \ddagger$ & $2.582 \ddagger$ & $2.585 \ddagger$ \\
\hline Paris & 0.079 & $0.246^{*}$ & $0.229^{*}$ & 0.047 \\
\hline Log(Price) & $-7.847 \ddagger$ & $-8.062 \ddagger$ & $-7.790 \ddagger$ & $-7.108 \ddagger$ \\
\hline $\log (\text { Price })^{*}($ Age- 20$) / 10$ & $-0.233 \ddagger$ & $-0.235 \ddagger$ & $-0.232 \ddagger$ & $-0.220 \ddagger$ \\
\hline Log(Price) ${ }^{*} \log ($ Income $)$ & $0.808 \ddagger$ & $0.822 \ddagger$ & $0.799 \ddagger$ & $0.746 \ddagger$ \\
\hline Number Railway stations & -0.0014 & -0.0032 & -0.0080 & 0.0053 \\
\hline Number Subway stations & $0.011^{*}$ & $0.011^{*}$ & -0.0013 & -0.0011 \\
\hline Average travel time from $\mathrm{j}$, commuting (TC) [hour] & $-0.251^{*}$ & -0.177 & $-0.493 \ddagger$ & $-0.635 \ddagger$ \\
\hline TC*(Dummy female) [hour] & -0.108 & -0.137 & -0.102 & -0.066 \\
\hline Average travel time from $\mathrm{j}$, by private car (VP) [hour] & -0.101 & 0.012 & 0.263 & 0.219 \\
\hline Distance to highway $\left[10^{3} \mathrm{~km}\right]$ & $-4.814^{*}$ & $-5.351^{*}$ & -2.922 & -3.238 \\
\hline$\%$ households with 1 member $* 1$ member in $\mathrm{h}$ & $3.246 \ddagger$ & $3.325 \ddagger$ & $3.660 \ddagger$ & $3.769 \ddagger$ \\
\hline$\%$ households with 2 members ${ }^{*} 2$ members in $\mathrm{h}$ & 0.137 & 0.257 & -0.082 & -0.591 \\
\hline$\%$ households with $3+$ members $^{*} 3+$ member in $\mathrm{h}$ & $2.962 \ddagger$ & $2.887 \ddagger$ & $2.627 \ddagger$ & $2.523 \ddagger$ \\
\hline$\%$ hh with no working memb. ${ }^{*}$ no working memb. in $\mathrm{h}$ & $6.384 \ddagger$ & $6.793 \ddagger$ & $6.940 \ddagger$ & $6.489 \ddagger$ \\
\hline$\%$ hh with 1 working memb. ${ }^{*} 1$ working memb. in $\mathrm{h}$ & -0.118 & 0.016 & 0.537 & 0.855 \\
\hline$\%$ hh with $2+$ working memb. ${ }^{*} 2+$ working memb. in $\mathrm{h}$ & -0.112 & -0.234 & $-0.758^{*}$ & $-0.890^{*}$ \\
\hline$\%$ poor households $*$ h poor & $1.148^{*}$ & $1.337 \dagger$ & $1.412 \dagger$ & $1.459 \dagger$ \\
\hline$\%$ households with a foreign head ${ }^{*}$ foreign head in $\mathrm{h}$ & $5.140 \ddagger$ & $4.628 \ddagger$ & $4.771 \ddagger$ & $5.125 \ddagger$ \\
\hline$\%$ households with a foreign head ${ }^{*}$ French head in $\mathrm{h}$ & $-3.849 \ddagger$ & $-4.327 \ddagger$ & $-4.026 \ddagger$ & $-3.575 \ddagger$ \\
\hline Employment $[1,000]$ & $1.951^{*}$ & $3.289 \ddagger$ & $3.913 \ddagger$ & 0.263 \\
\hline Density (Population/Surface) [1000 persons $/ \mathrm{km}]$ & 0.661 & 2.167 & 3.079 & $-6.625^{*}$ \\
\hline Log(Population) & $0.211 \ddagger$ & $0.377 \ddagger$ & $0.728 \ddagger$ & $1.011 \ddagger$ \\
\hline \% change in population, 1990 to 1999 & $0.201 \dagger$ & $0.232 \ddagger$ & $0.185^{*}$ & $0.185 \dagger$ \\
\hline${\text { Pseudo } \mathrm{R}^{2}}^{2}$ & $14.4 \%$ & $15.7 \%$ & $21.3 \%$ & $30.6 \%$ \\
\hline
\end{tabular}

*: significant at the $10 \%$ level; $\uparrow$ : significant at the $5 \%$ level; $\ddagger$ : significant at the $1 \%$ level

The first result to be noticed is that the variations in the coefficients as a function of the

\footnotetext{
${ }^{7}$ In this case, repetitions of the same dwelling in the same individual choice set should be excluded, but the probability of repetition in a set of more than 5 million dwellings is negligible.
} 
number of alternatives are very limited for the coefficients which are significant. This is consistent with the theory that importance sampling leads to unbiased coefficients when a correcting term (size variable, expressed in log terms) is introduced in the model. ${ }^{8}$ On the opposite, the unsignificant coefficients tend to be very unstable (e.g. number of stations, commuting travel time for women and travel time or distance to highway). This is in agreement with expectations and probably results from the large correlation between these variables. However, the impact of commuting travel time becomes significant in larger samples. The coefficient of the " $\log$ (Population)" significantly varies as a function of the number of alternatives, which is consistent with the fact that, as explained in footnote 8 , it reflects both a correction term (heavily dependent on the number of sampled alternatives) term and preferences.

The second result to be noticed is that the explanatory power dramatically increases with the number of alternatives (from $14.4 \%$ to $30.6 \%$ ). This is consistent with the fact that the coefficient of the size term " $\log ($ Population)" becomes larger and more significant, suggesting that this variable is a far better proxy for the list of commune dummies when the number of alternatives increases.

\subsection{Location choice with capacity constraints}

\subsubsection{Estimation of a more parsimonious model with and without constraints}

In order to estimate the effect of the availability constraints based on the yearly demand, it is necessary to infer an annual supply of dwellings from the current supply observed in March 1999. This annual supply depends on the Housing Turnover Index $\lambda$ defined in Section 2.3.

As a preliminary sensitivity analysis, we consider two cases: $\lambda=0.5$ and $\lambda=0.45$. Recall that these values correspond to an upper bound for the HTI, that is, a lower bound for the constraints. The results obtained when estimating a more parsimonious model (with only 5 explanatory variables) on a $2 \%$ sample of households with all alternatives are reported in Table 6. ${ }^{9}$ The "initial" estimates corresponds to the model estimated assuming that the system is not constrained (iteration $l^{\prime}=0$ in Algorithm U, see Appendix), whereas the "final" estimates correspond to (iteration $l^{\prime}=\infty$ in Algorithm U).

Since random sampling introduces heterogeneity in the individual allocation ratios $\Omega^{i}$, the homogeneous model can only be estimated using the universal set of alternatives (1,300 municipalities). However, the data set with universal choice set and $100 \%$ of the movers would contain 766 million lines and its size would be over 2 terabytes with all the variables used in the previous section! In order to illustrate the method on a more tractable data set, we used a representative random sample of $2 \%$ households, and we selected a small number of explanatory variables

\footnotetext{
${ }^{8}$ In the case of importance sampling (an alternative is included in the choice set with a probability proportional to the number of households which have chosen this alternative), the coefficient of this correcting term should be equal to 1 (see [3]). However, the size variable can also enter preferences. Its coefficient can therefore be estimated freely, since it merges preferences, fitting considerations (see above) and correcting factor. Note that we did not consider here exactly a size variable (number of dwellings) but instead a population variable. The difference between these two variables is the log of average number of household members, which may also enter preferences. Since all these variables are highly correlated between each other and with other explanatory variables, they cannot be introduced simulatenously in the model, and we selected the most significant set of variables.

${ }^{9}$ The dataset size would prevent from estimating the full model on the same sample since four times more variables roughly means four times more RAM necessary.
} 
(Log(Price); Average travel time from $j$, commuting (TC); \% households with a foreign head; $\log ($ Population); and Same district as before move in the heterogeneous case). This selection is based on results obtained in the previous sections and on other results not reported here in order to save space. With this parsimonious model, the explanatory power is still reasonable in the heterogeneous case, and sample size is tractable $(15,321,800$ lines, 11, 786 movers and slightly more than $1 \mathrm{~Gb}$ ). Although convergence is attained after a small number of iterations (less than 5 iterations for constraints at each estimation loop, less than 10 demand loops), about 2 hours are necessary for estimating the homogeneous version of the model, and computing time goes over 15 hours for the heterogeneous version model. ${ }^{10}$ In the future, working on a more powerful computer and improving the efficiency of the algorithm will allow increasing significantly both the sampling rate and the number of explanatory variables. A trade-off between the number of households, the number of alternatives by individual and the number of explanatory variables will remain, however.

The overall fit of this parsimonious model is quite good given the low number of explanatory variables: in the $2 \%$ sample with all alternatives, the Pseudo- $\mathrm{R}^{2}$ is nearly $30 \%$ in the heterogeneous case and $23 \%$ in the homogeneous case. The unexpected positive sign of the price coefficient reflects an omission bias: some alternative attributes (e.g. \% poor households) are correlated both with price and with demand. Because of this omission bias (which is corrected by the introduction of additional explanatory variables, as in the full model), we will not interpret the value of the price coefficient. The extreme sensitivity of the price coefficient to omission bias has already been noticed by [8]. In addition, results not reported here show that the price coefficient value and significance level are very different in a $1 \%$ sample of households, and even more different in the full model. The most significant variable remains the dummy "same district as before move", and the corresponding estimated coefficient is stable across sample sizes. Strangely enough, in this parsimonious specification, the price coefficient is unchanged when the factor correcting for constraints is introduced.

We could anticipate identification problems because both price and correcting factor $\log \left(\pi_{j}^{i}\right)$ reflect the supply/demand ratio in some sense: $\pi_{j}^{i}=S_{j} / D_{j}$ in the alternatives constrained ex post and equilibrium on the dwelling market suggests that price depends on the supply/demand ratio. However, the correlation between $\log$ (Price) and supply/demand ratio is only $-4.85 \%$ (significant at the $10 \%$ level but not at the $5 \%$ level) for initial demand estimates and $-1.12 \%$ (not significant at all) for final demand estimates, suggesting that prices are only marginally influenced by the frictions in the housing market. In other words, prices do not actually clear the market, a conclusion we can only generalize at this point to the Ile-de-France housing market. Note that the price coefficient is not significant in the $1 \%$ sample and only marginally significant in the $2 \%$ sample. However, the price coefficient becomes more significant in larger samples and negative when enough covariates are added, as in the full model. In the parsimonious model, the coefficients of the accessibility variable (average travel time from the commune) and of the size variable $(\log$ (Population) ) are stable across sample sizes and between initial and final estimates. By contrast, the fraction of foreign households is more sensitive both to the sample size and to the introduction of constraints.

\footnotetext{
${ }^{10}$ Using the SAS software on an IBM laptop with 2 Gb RAM.
} 
Table (6): Comparison of results in the homogeneous and heterogeneous cases (2\% sample)

\begin{tabular}{|c|c|c|c|}
\hline \multicolumn{2}{|l|}{ Variable } & $\begin{array}{l}\text { Homogeneous case } \\
\text { Coefficient (t-stat) }\end{array}$ & $\begin{array}{l}\text { Heterogeneous case } \\
\text { Coefficient (t-stat) }\end{array}$ \\
\hline \multirow{4}{*}{ Same district as before move } & initial, $\lambda=0.45$ & does not apply & $2.5765(105.71)$ \\
\hline & final, $\lambda=0.45$ & does not apply & $2.5890(106.05)$ \\
\hline & initial, $\lambda=0.5$ & does not apply & $2.5765(105.71)$ \\
\hline & final, $\lambda=0.5$ & does not apply & $2.5800(105.93)$ \\
\hline \multirow{4}{*}{$\log ($ Price $)$} & initial, $\lambda=0.45$ & $0.0280(1.67)$ & $0.0219(1.30)$ \\
\hline & final, $\lambda=0.45$ & $0.0321(1.92)$ & $0.0268(1.59)$ \\
\hline & initial, $\lambda=0.5$ & $0.002501(0.11)$ & $0.0219(1.30)$ \\
\hline & final, $\lambda=0.5$ & $0.000250(0.01)$ & $0.0260(1.54)$ \\
\hline \multirow{4}{*}{ Average travel time from $\mathrm{j}$} & initial, $\lambda=0.45$ & $-0.2443(-2.27)$ & $-0.2876(-2.64)$ \\
\hline & final, $\lambda=0.45$ & $-0.1392(-1.28)$ & $-0.1999(-1.82)$ \\
\hline & initial, $\lambda=0.5$ & $-0.3170(-2.07)$ & $-0.2876(-2.64)$ \\
\hline & final, $\lambda=0.5$ & $-0.3302(-2.14)$ & $-0.2567(-2.34)$ \\
\hline \multirow{4}{*}{$\%$ hh with a foreign head } & initial, $\lambda=0.45$ & $-0.4483(-2.28)$ & $-0.5612(-2.77)$ \\
\hline & final, $\lambda=0.45$ & $-0.8548(-4.21)$ & $-0.9530(-4.57)$ \\
\hline & initial, $\lambda=0.5$ & $-0.9955(-3.46)$ & $-0.5612(-2.77)$ \\
\hline & final, $\lambda=0.5$ & $-1.0966(-3.71)$ & $-0.8345(-4.05)$ \\
\hline \multirow{4}{*}{ Log(Population) } & initial, $\lambda=0.45$ & $1.0846(105.27)$ & $1.0722(100.23)$ \\
\hline & final, $\lambda=0.45$ & $1.0590(98.82)$ & $1.0495(94.76)$ \\
\hline & initial, $\lambda=0.5$ & $1.0807(105.27)$ & $1.0722(100.23)$ \\
\hline & final, $\lambda=0.5$ & $1.0733(68.33)$ & $1.0496(94.23)$ \\
\hline \multirow{2}{*}{$\log \left(\pi_{j}^{i}\right)$} & final, $\lambda=0.45$ & $0.5157(8.27)$ & $0.3888(7.88)$ \\
\hline & final, $\lambda=0.5$ & $0.7848(7.22)$ & $0.7848(7.22)$ \\
\hline \multirow{4}{*}{ Pseudo-R ${ }^{2}$} & initial, $\lambda=0.45$ & $22.90 \%$ & $29.68 \%$ \\
\hline & final, $\lambda=0.45$ & $22.94 \%$ & $29.72 \%$ \\
\hline & initial, $\lambda=0.5$ & $23.15 \%$ & $29.84 \%$ \\
\hline & final, $\lambda=0.5$ & $23.19 \%$ & $29.92 \%$ \\
\hline
\end{tabular}

We are also interested in the sensitivity of the results to the sampling ratio and compare the results obtained on a $1 \%$ and on a $2 \%$ representative samples of households. Because of constraints on the data sets size, we only explore the parsimonious models (see Table 8). The "Same district as before move" dummy is used in the heterogeneous case, but not in the homogeneous one.

\subsubsection{Constraints and allocation ratio in the parsimonious model}

We turn now to an assessment in the homogeneous case (parsimonious model) of the effect, on the fraction of alternatives constrained and on the allocation ratio, of the sampling rate of households and of $\lambda$. Table 7 presents the results for $1 \%$ and $2 \%$ samples of households, using $\lambda=0.5$ and $\lambda=0.45$.

Table (7) Fraction alternatives constrained and allocation ratio, homogeneous case 


\begin{tabular}{|c|l|l|l|}
\hline & $\lambda=0.5,1 \%$ & $\lambda=0.5,2 \%$ & $\lambda=0.45,2 \%$ \\
\hline \% alt. constrained: & & & \\
Ex ante, initial demand estimates & $36.2 \%$ & $40.2 \%$ & $47.31 \%$ \\
Ex post, initial demand estimates & $39.3 \%$ & $43.5 \%$ & $52.69 \%$ \\
Ex ante, final demand estimates & $46.5 \%$ & $51.5 \%$ & $61.77 \%$ \\
Ex post, final demand estimates & $51.2 \%$ & $56.2 \%$ & $70.85 \%$ \\
\hline Average [min;max] $S_{j} / D_{j}$ ratio: & & & \\
Initial demand estimates & $1.18[0.06 ; 5.48]$ & $1.14[0.06 ; 4.97]$ & $1.09[0.06 ; 4.94]$ \\
Final demand estimates & $1.08[0.01 ; 5.26]$ & $1.03[0.03 ; 4.64]$ & $0.91[0.00 ; 4.53]$ \\
\hline Allocation ratio $\Omega:$ & & & \\
Ex ante, initial demand estimates & 1.019 & 1.021 & 1.037 \\
Ex post, final demand estimates & 1.050 & 1.039 & 1.127 \\
\hline
\end{tabular}

For all values of the HTI $\lambda$, we have the following result: the fraction of constrained alternatives is always larger once algorithm $\mathrm{K}$ has converged. This is true when the initial demand is considered and when the final demand is considered. For the final demand, the fraction of constrained alternatives is (almost always) larger than for the initial demand at any stage of algorithm K. In other words, convergence of the inner or outer loop increases the fraction of constrained alternatives. The reason behind this intuition is as follows: at the initial demand loop, the observed demand under-estimates the ex ante demand in constrained alternatives and over-estimates ex ante demand in unconstrained alternatives. These biases are corrected at the final demand loop. As expected, ceteris paribus, when $\lambda$ decreases, the level of constraints increases since a lower value of $\lambda$ means a smaller number of dwellings available on average during one year. Note that a very small variation of $\lambda$ induces an important variation of the fraction of alternatives constrained.

The average ratio of supply to demand, which is inversely proportional to the degree of constraint, decreases from the initial demand to the final demand (since this increases the amount of constraints). The same reasoning applies to $\Omega$, which is an increasing function of the level of constraints. According to the final estimates, when $\lambda=0.5,4 \%$ to $5 \%$ of the households are not located in their preferred commune.

Similar results are obtained in the heterogeneous case, with the parsimonious model. The results (not reported here) for the heterogeneous case are qualitatively similar to the ones of the homogeneous case in terms of the fraction of alternatives constrained and the supply over demand ratio. Note that the allocation ratio in the heterogeneous case is specific either to the household or to the alternative. When $\lambda=0.5$, the average individual-specific allocation ratio is approximately equal to the homogeneous allocation ratio, but this is no more true when $\lambda=0.45$. In that case, the fraction of households not located in their preferred commune increases to $13 \%$ when $\lambda=0.45$.

\subsubsection{Extent of the constraints and allocation ratio in the full model (heterogen- eous case)}

Here we examine, in the heterogeneous case, the effect on the fraction of alternatives constrained and on the allocation ratio of the sampling rate of households and of alternatives. Table 8 
presents the results in the case when demand is estimated using the full model (with 24 explanatory variables) and the HTI $\lambda$ is specific to the district. Note that constraints are more severe in that case because the average HTI used here (see Section 2.3) is 39\% for Ile-de-France. This new set of results should therefore be considered as an upper bound for the constraints (since it is based on a lower bound for HTI).

The model could not be estimated on the 1300 alternatives/0.5\% sample because it ran out of memory after the estimation of the initial demand. The results are significantly different from those obtained in the previous section, and are highly sensitive to the number of alternatives used for the estimation. The fraction of alternatives constrained ex ante with initial demand estimates is also sensitive to the sampling ratio, but the fraction of alternatives constrained ex post with final demand is less sensitive to the sampling ratio. However, the constraints are not very severe in most of these alternatives, and the average allocation ratio remains close to 1.13 ex ante and 1.30 ex post in all sample sizes with 100 alternatives, which means that $30 \%$ of households are not located in their preferred commune.

Table (8) Fraction alternatives constrained and allocation ratio, heterogeneous case, districtspecific HTI

\begin{tabular}{|c|c|c|c|c|c|c|c|c|}
\hline $\begin{array}{c}\text { Nb.alt. } \\
\text { sampling rate }\end{array}$ & $\begin{array}{c}500 \\
0.5 \%\end{array}$ & $\begin{array}{c}200 \\
0.5 \%\end{array}$ & $\begin{array}{c}100 \\
0.5 \%\end{array}$ & $\begin{array}{l}100 \\
1 \%\end{array}$ & $\begin{array}{l}100 \\
2 \%\end{array}$ & $\begin{array}{l}100 \\
3 \%\end{array}$ & $\begin{array}{l}100 \\
4 \%\end{array}$ & $\begin{array}{l}100 \\
5 \%\end{array}$ \\
\hline $\begin{array}{l}\% \text { alt. constr.: } \\
\text { ex ante, initial }\end{array}$ & $27.0 \%$ & $29.5 \%$ & $40.3 \%$ & $38.7 \%$ & $43.8 \%$ & $38.9 \%$ & $38.2 \%$ & $40.9 \%$ \\
\hline ex post, initial & $43.7 \%$ & $50.8 \%$ & $57.7 \%$ & $59.2 \%$ & $63.0 \%$ & $58.5 \%$ & $59.2 \%$ & $60.4 \%$ \\
\hline ex ante, final & $34.8 \%$ & $37.7 \%$ & $45.8 \%$ & $44.2 \%$ & $48.5 \%$ & $41.8 \%$ & $43.0 \%$ & $46.2 \%$ \\
\hline ex post, final & $62.5 \%$ & $69.1 \%$ & $67.9 \%$ & $68.8 \%$ & $75.5 \%$ & $69.2 \%$ & $71.7 \%$ & $73.8 \%$ \\
\hline $\begin{array}{c}\text { Average } \Omega^{i} \text { : } \\
\text { ex ante, initial }\end{array}$ & 1.167 & 1.179 & 1.138 & 1.130 & 1.136 & 1.134 & 1.129 & 1.131 \\
\hline ex post, final & 1.478 & 1.444 & 1.314 & 1.302 & 1.311 & 1.303 & 1.303 & 1.321 \\
\hline $\begin{array}{l}{[\min ; \max ] \Omega^{i} \text { : }} \\
\text { ex ante, initial }\end{array}$ & {$[1.07 ; 1.35]$} & {$[1.06 ; 1.55]$} & {$[1.02 ; 1.74]$} & {$[1.02 ; 1.86]$} & {$[1.01 ; 2.06]$} & {$[1.01 ; 2.54]$} & {$[1.01 ; 1.95]$} & {$[1.01 ; 1.95]$} \\
\hline ex post, final & {$[1.00 ; 3.70]$} & {$[0.91 ; 5.35]$} & {$[0.87 ; 5.25]$} & {$[0.83 ; 7.38]$} & {$[0.61 ; 8.02]$} & {$[0.23 ; 13.4]$} & {$[0.44 ; 9.15]$} & {$[0.42 ; 12.3]$} \\
\hline $\begin{array}{c}\text { Average } \bar{\Omega}_{j} \text { : } \\
\text { ex ante, initial }\end{array}$ & 1.194 & 1.199 & 1.159 & 1.157 & 1.169 & 1.157 & 1.153 & 1.156 \\
\hline ex post, final & 1.717 & 1.636 & 1.441 & 1.442 & 1.472 & 1.431 & 1.445 & 1.482 \\
\hline $\begin{array}{l}{[\min ; \max ] \bar{\Omega}_{j} \text { : }} \\
\text { ex ante, initial }\end{array}$ & {$[1.13 ; 1.25]$} & {$[1.08 ; 1.41]$} & {$[1.03 ; 1.55]$} & {$[1.08 ; 1.43]$} & {$[1.09 ; 1.39]$} & {$[1.09 ; 1.34]$} & {$[1.08 ; 1.33]$} & {$[1.10 ; 1.31]$} \\
\hline ex post, final & {$[1.23 ; 2.43]$} & {$[-; 3.06]$} & {$[-; 4.49]$} & {$[1.08 ; 3.45]$} & {$[1.11 ; 2.35]$} & {$[1.14 ; 2.56]$} & {$[1.14 ; 2.36]$} & {$[1.16 ; 3.0$} \\
\hline
\end{tabular}

\subsubsection{Comparison of constrained and unconstrained aggregate demand}

We now turn to the aggregate results and compare the demand predicted by the different models in each commune to actual (observed) demand, that is the number of movers observed in 1998. At a first glance, the correlations between aggregate demand $\left(D_{j}\right)$ estimated in the different specifications and samples is very high. However, the precision of aggregate demand deteriorates in small municipalities when sample size becomes too small. 
Table 9 reports the correlation coefficient between the actual demand (number of households which moved in to each commune in 1998) and the demand predicted by the different models. The "Full model" was estimated using a random sample of 8 alternatives for each household, with 24 explanatory variables, as reported in Table 5. The "Parsimonious model" was estimated using the same random sampling of 8 alternatives, but with only 5 explanatory variables, as reported in Table 6 . The estimates with constraints are those of the homogeneous case with $\lambda=0.5$. Table 9 shows that the accuracy of the aggregate estimates remains satisfactory when the sampling ratio decreases. It is interesting to notice that accuracy is not significantly altered in the parsimonious model. It seems that the accuracy becomes poorer and poorer (correlation less than $90 \%$ in the $1 \%$ sample, less than $93 \%$ in the $2 \%$ sample) when the "size" (measured as the number of observed moves into the commune in 1998) of the alternatives decreases. The correlation with actual demand remains quite high in the 1148 municipalities with less than 1000 movers in 1998, but it diminishes in the 1054 municipalities with less than 500 movers in 1998 and becomes even lower in smaller alternatives. However, accuracy significantly improves in the $1 \%$ and $2 \%$ samples of households when a universal set of alternatives is used for the demand predictions. Note that with a universal set of alternatives the accuracy is the same in the $1 \%$ and $2 \%$ samples of households, whereas, with a random sampling of 8 alternatives, accuracy was far poorer in the $1 \%$ sample compared to the $2 \%$ sample of households (in the small municipalities). Integrating the constraints significantly improves the accuracy of the demand estimates, especially for small alternatives.

Table (9): Correlation between actual number of movers and demand predicted by different models

\begin{tabular}{|l|l|c|c|c|c|c|}
\hline Model / sampling rate / Nb alt. & All alt. & $\begin{array}{c}<1000 \\
1148\end{array}$ & $\begin{array}{c}<500 \\
1054\end{array}$ & $\begin{array}{c}<200 \\
948\end{array}$ & $\begin{array}{c}<100 \\
841\end{array}$ & $\begin{array}{c}<50 \\
725\end{array}$ \\
\hline Full / 100\% / 8 & 0.9979 & 0.9941 & 0.9893 & 0.9764 & 0.9599 & 0.9205 \\
\hline Parsimonious / 100\% / 8 & 0.9968 & 0.9877 & 0.9765 & 0.9626 & 0.9444 & 0.8979 \\
\hline Full / 20\% / 8 & 0.9979 & 0.9919 & 0.9855 & 0.9672 & 0.9329 & 0.8726 \\
\hline Parsimonious / 20\% / 8 & 0.9967 & 0.9864 & 0.9726 & 0.9535 & 0.9206 & 0.8595 \\
\hline Full / 2\% / 8 & 0.9968 & 0.9735 & 0.9398 & 0.8701 & 0.7441 & 0.6258 \\
\hline Parsimonious / 2\% / 8 & 0.9958 & 0.9702 & 0.9361 & 0.8878 & 0.7775 & 0.6702 \\
\hline Full model, 1\% / 8 & 0.9958 & 0.9554 & 0.8952 & 0.7659 & 0.6180 & 0.5124 \\
\hline Parsimonious / 1\% / 8 & 0.9950 & 0.9531 & 0.8986 & 0.7935 & 0.6563 & 0.5529 \\
\hline Parsimonious / 2\% / all & 0.9953 & 0.9803 & 0.9635 & 0.9427 & 0.9218 & 0.8582 \\
\hline Parsimonious / 2\% / all, constraints & 0.9955 & 0.9834 & 0.9722 & 0.9625 & 0.9426 & 0.9005 \\
\hline Parsimonious / 1\% / all & 0.9953 & 0.9799 & 0.9624 & 0.9397 & 0.9176 & 0.8582 \\
\hline Parsimonious / 1\% / all, constraints & 0.9997 & 0.9828 & 0.9712 & 0.9610 & 0.9413 & 0.9005 \\
\hline
\end{tabular}

\section{Conclusion and extensions}

Constraints on the availability of alternatives have been demonstrated for the housing market in the Ile-de-France. We have shown that housing prices do tend to capitalize the imbalances between demand and supply in the short run period of one year, but do not fully accomplish the task of resolving short-term market disequilibrium. The specification linking residential location 
choices and housing prices through the aggregation of individual demands provides a tractable means to address this economic endogeneity issue, and our results demonstrate that the market clearing role for prices is significant. However, even after accounting for the price effect, a large fraction of alternatives still had excess demand, imposing availability constraints on consumers that ultimately must make suboptimal choices from unconstrained alternatives. This is a significant empirical finding, since virtually all previous literature in discrete choice modeling in market contexts implicitly or explicitly assume that prices clear the market. We note that excess demand appears to be more significant when we consider hererogeneity introduced by including interactions of household and alternative characteristics in the utility function. This suggests that more complete specifications of the interactive factors influencing utility are more likely to explain the uneven distributions of demands of different types of housing consumers, and result in more accurate estimation of the excess demand.

We have developed and demonstrated a viable mechanism to address the influence of constrained alternatives on choice outcomes, by using relatively weak assumptions consistent with the property of independence of irrelevant alternatives inherent in the multinomial logit model. Basically, we assume that (1) if an individual prefers an alternative $j$, which is unconstrained ex post, he can be sure to be allocated to it, and (2) in an alternative constrained ex post, the individual allocation ratio is the same for all individuals. We then develop a discrete choice model specification and computational algorithm that corrects for capacity constraints in a way that is consistent with the IIA assumption. In can be shown that this algorithm converges to a unique equilibrium in the case of homogeneous agents. Though we have not proven the existence and uniqueness of an equilibrium solution in the heterogeneous case, simulation results suggest that convergence and uniqueness occur. We leave the theoretical proofs for future research.

Application of this constrained discrete choice algorithm confirms that the share of alternatives initially estimated to be constrained significantly increases once the parameter estimates are adjusted to correct for the effect of the constraints. In our heterogeneous case, the initial estimate of ex ante constraints was that $30 \%$ to $40 \%$ of communes were constrained, depending on sampling rates. At convergence of the algorithm, after accounting for the complex spillovers of households forced to make second-best choices among initially unconstrained alternatives that could become constrained due to these spillovers, this estimate increased to $62 \%$ to $75 \%$.

Our sensitivity testing confirms that most parameter estimates in the unconstrained model are fairly robust to sampling of households, down to quite small proportions such as 1 to $2 \%$, where precision decreases. Due to the size of the data and the use of a full enumeration of 1300 alternatives in the heterogeneous case, we were limited to using small sampling rates for households for testing a very parsimonious model that clearly reflects omitted variables bias, but allowed the initial testing of the algorithm to proceed. The results of this testing confirm that the algorithm converges after a small number of iterations, and that parameters can be adjusted for the bias resulting from capacity constraints. Finally, our analysis of demand predictions confirms that the constrained discrete choice algorithm significantly improves predictions of demand, particularly for those alternatives with relatively small market share. This may have important implications for research in which the alternatives of interest reflect small market shares, and the system contains some capacity constraints - even if the alternatives of interest are not themselves constrained ex ante.

The method developed so far can be improved along several directions. First, our results 
are sensitive to the value of the Housing Turnover Index $\lambda$, which could be estimated precisely on our data. A more behavioral approach could help deriving the individual optimal search time (which is implicitly aggregated in our HTI $\lambda$ ), following an approach similar to the one used in [4] in the case of automobile market. Second, other allocation mechanisms could be envisaged. Our allocation mechanism relies to a certain extent on an assumption of equity in the adjustment of choice probabilities. Finally, further empirical testing and refinement of the computational performance of the constrained choice algorithm, and implementation in generally-available software, remain to be completed, and these steps will make it possible to explore applications of this approach to policy questions relevant to constrained choice contexts.

\section{Acknowledgements}

This research has been funded in part by PREDIT, the French Department of Transportation (contract awarded to IAURIF, University of Cergy-Pontoise and adpC). We would like to thank Dany Nguyen (IAURIF) for providing us with the data for the Ile-de-France region and Kiarash Motamedi for helping us handling this data. We benefited from the comments of the seminar participants of the second Khumo conference (in particular from Esko Niskanen, Edoardo Marcucci, Robin Lindsey and Stef Proost), of the Dijon conference organized by Alban Thomas, and from the Fiesta conference participants at the Ecole Nationale des Ponts et Chaussées (in particular Dominique Schwartz and Joël Maurice). We would like also to thank the participants of the seminar organized in the honour of Ken Small, especially Chen Hsiao and Ken Train for extremely helpful comments and suggestions, and participants to the Regional Science Association meeting, Totonto, 2006, in particular Jan Rouwendhal. The sampling methods were discussed with Daniel McFadden and Moshe Ben-Akiva, who provided us with valuable advice. The third author wishes to acknowledge the National Science Foundation Grants EIA-0121326 and IIS-0534094, and Environmental Protection Agency grant EPA R831837. Finally, we would like to thank two anonymous referees and the editor Jan Brueckner for their useful comments and suggestions.

\section{References}

[1] A. Anas and J. Cho. A dynamic, policy oriented model of the regulated housing market; the swedish prototype. Regional Science Urban Economics, 18:210-231, 1988.

[2] S. Anderson, A. de Palma, and J. Thisse. Discrete Choice Theory of Product Differentiation. MIT Press, 1993.

[3] M. Ben-Akiva and S. R. Lerman. Discrete Choice Analysis: Theory and Application to Travel Demand. MIT Press, 1985.

[4] S. Berry, J. Levinsohn, and A. Pakes. Automobile prices in market equilibrium. Econometrica, 63(4):841-890, July 1995.

[5] A. de Palma and C. Lefèvre. A probabilistic search model. Journal of Mahematical Sociology, 8:43-60, 1981. 
[6] A. de Palma, K. Motamedi, N. Picard, and P. Waddell. A model of residential location choice with endogenous housing prices and traffic for the paris region. European transport, 2006 .

[7] A. de Palma and J. Rouwendal. Availability constraints in the housing market. Journal of housing economics, 5:105-132, 1996.

[8] C. A. Guevara and M. Ben-Akiva. Endogeneity in residential location choice models. Unpublished manuscript, 2006.

[9] S. Lerman. Location, housing, automobile ownership, and the mode to work: A joint choice model. Transportation Research Board Record, 610:6-11, 1977.

[10] J. Louviere, K. Train, M. Ben-Akiva, C. Bhat, A. A. C. David Brownstone, R. T. Carson, J. R. Deshazo, D. Fiebig, W. Greene, D. Hensher, and D. Waldman. Recent progress on endogeneity in choice modeling. Marketing Letters, 16(3-4):255-265, 2005. Special Issue: Sixth Invitational Choice Symposium.

[11] D. McFadden. Modelling the choice of the residential location. In A. Karlqvist, L. Lundqvist, F. Snickars, and J. Weibull, editors, Spatial Interaction Theory and Residential Location, pages 75-96. North Holland, Amsterdam, 1978.

[12] J. Quigley. Housing demand in the short-run: An analysis of polytomous choice. Explorations in Economic Research, 3:76-102, 1976.

[13] G. Weisbrod, S. Lerman, and M. Ben-Akiva. Tradeoffs in residential location decisions: Transportation versus other factors. Transport Policy and Decision Making, 1:13-26, 1980.

[14] R. C. J. Williams. A logit model of demand for neighborhood. In D. Segal, editor, The Economics of Neighborhood, pages 17-42. Academic Press, New York, 1979.

\section{Appendix}

\section{Algorithm K: known demand}

1. Check that aggregate demand is smaller than aggregate supply (see Condition (4)).

2. Iteration $l=0$ (initialization): set $\Omega^{i}=1$; compute the set $\mathcal{C}(0)=\left\{j \in \mathcal{J} \mid S_{j} \leq D_{j}\right\}$ of alternatives constrained at iteration zero (that is, constrained ex ante). The set of alternatives unconstrained at iteration zero (that is, unconstrained ex ante) is given by: $\overline{\mathcal{C}}(0)=\mathcal{J} \backslash \mathcal{C}(0)$.

3. Compute the individual allocation ratios $\Omega^{i}(0)=\frac{1-\sum_{j \in \mathcal{C}(0)} \frac{S_{j}}{D_{j}} \mathbb{P}_{j}^{i}}{1-\sum_{j \in \mathcal{C}(0)} \mathbb{P}_{k}^{i}}$, using Lemma 3. 
4. Compute the alternative-specific allocation ratios using Equation (9):

$$
\bar{\Omega}_{j}(0)=\frac{\sum_{i=1}^{N} \Omega^{i}(0) \mathbb{P}_{j}^{i}}{\sum_{i=1}^{N} \mathbb{P}_{j}^{i}} .
$$

5. Update iteration: $l \rightarrow l+1$. Update the constrained choice set using Condition (10) and Definition (9):

$$
\mathcal{C}(l+1)=\left\{j \in \mathcal{J} \mid S_{j} \leq \bar{\Omega}_{j}(l) D_{j}=\sum_{i=1}^{N} \Omega^{i}(l) \mathbb{P}_{j}^{i}\right\}
$$

6. Update: $\Omega^{i}(l+1)=\frac{1-\sum_{j \in \mathcal{C}(l+1)} \frac{S_{j}}{D_{j}} \mathbb{P}_{j}^{i}}{\sum_{k \in \overline{\mathcal{C}}(l+1)} \mathbb{P}_{k}^{i}}$

7. Update $\bar{\Omega}_{j}(l+1)=\frac{\sum_{i=1}^{N} \Omega^{i}(l+1) \mathbb{P}_{j}^{i}}{\sum_{i=1}^{N} \mathbb{P}_{j}^{i}}$

8. Stop at iteration $l+1$ if $\mathcal{C}(l+1)=\mathcal{C}(l)$ (and therefore $\Omega^{i}(l+1)=\Omega^{i}(l)$ for all $\left.i=1 \ldots N\right)$, else go to 5 . By definition, convergence is attained at $l=\infty$.

9. Compute the correction factor

$$
\ln \left(\pi_{j}^{i}\right)=\left\{\begin{array}{c}
\ln \left(S_{j} / D_{j}\right) \text { if } j \in \mathcal{C} \\
\ln \left(\Omega^{i}(\infty)\right) \text { if } j \in \overline{\mathcal{C}}
\end{array} .\right.
$$

using Theorem 1.

In the homogenous case, it can be shown that this algorithm converges. Simulation experiments suggest that it also converge in the heterogenous case.

\section{Algorithm U: unknown demand}

1. Check that the aggregate demand is smaller than the aggregate supply (Condition (4)).

2. Iteration $l^{\prime}=0$ (initialization of demand of the estimation module): estimate a MNL model assuming initially no constraints (i.e. $\Omega^{i}=\bar{\Omega}_{j}=\pi_{j}^{i}=1$ ). This leads to the estimated choice probabilities $\hat{\mathbb{P}}_{j}^{i}(0)$ and to the estimated demand $\hat{D}_{j}(0)=\sum_{i=1}^{N} \hat{\mathbb{P}}_{j}^{i}(0)$. The correcting factors $\ln \left[\pi_{j}^{i}(0)\right]$ are initialized to 0 and play no role in Equation (11a).

(a) Iteration $l=0$ (initialization of the constraints): compute the set

$$
\mathcal{C}(0,0)=\left\{j \in \mathcal{J} \mid S_{j} \leq \hat{D}_{j}(0)\right\}
$$

of alternatives constrained ex ante for the initial estimated demand, using Condition (10). 
(b) Compute the individual allocation ratio, using Lemma 3:

$$
\Omega^{i}(0,0)=\left(1-\sum_{j \in \mathcal{C}(0,0)} \frac{S_{j}}{\hat{D}_{j}(0)} \hat{\mathbb{P}}_{j}^{i}(0)\right) /\left(1-\sum_{j \in \mathcal{C}(0,0)} \hat{\mathbb{P}}_{j}^{i}(0)\right) .
$$

(c) Compute the alternative-specific allocation ratio using Equation (9):

$$
\bar{\Omega}_{j}(0,0)=\left(\sum_{i=1}^{N} \Omega^{i}(0,0) \hat{\mathbb{P}}_{j}^{i}(0)\right) /\left(\sum_{i=1}^{N} \hat{\mathbb{P}}_{j}^{i}(0)\right) .
$$

(d) Update iteration for algorithm $\mathrm{K}: l \rightarrow l+1$. Update the set of alternatives constrained using Condition (10):

$$
\mathcal{C}(0, l+1)=\left\{j \in \mathcal{J} \mid S_{j} \leq \bar{\Omega}_{j}(0, l) \hat{D}_{j}(0)=\sum_{i=1}^{N} \Omega^{i}(0, l) \hat{\mathbb{P}}_{j}^{i}(0)\right\}
$$

(e) Update $\Omega^{i}(0, l+1)=\left(1-\sum_{j \in \mathcal{C}(0, l+1)} \frac{S_{j}}{\hat{D}_{j}(0)} \hat{\mathbb{P}}_{j}^{i}(0)\right) /\left(1-\sum_{j \in \mathcal{C}(0, l+1)} \hat{\mathbb{P}}_{j}^{i}(0)\right)$

(f) Update $\bar{\Omega}_{j}(0, l+1)=\left(\sum_{i=1}^{N} \Omega^{i}(0, l+1) \hat{\mathbb{P}}_{j}^{i}(0)\right) /\left(\sum_{i=1}^{N} \hat{\mathbb{P}}_{j}^{i}(0)\right)$.

(g) Stop at iteration $l+1$ if $\mathcal{C}(0, l+1)=\mathcal{C}(0, l)$ (and therefore $\Omega^{i}(0, l+1)=\Omega^{i}(0, l), i=$ $1 \ldots N)$, else go to step d.

(h) When convergence is attained $(l=\infty)$, we have $\mathcal{C}(0) \stackrel{\text { def }}{=}\left\{j \in \mathcal{J} \mid S_{j} \leq \bar{\Omega}_{j}(0, \infty) \hat{D}_{j}(0)\right\}$, $\Omega^{i}(0) \stackrel{\text { def }}{=} \Omega^{i}(0, \infty)$, and $\bar{\Omega}_{j}(0) \stackrel{\text { def }}{=} \bar{\Omega}_{j}(0, \infty)$.

3. Update iteration of the estimation module: $l^{\prime} \rightarrow l^{\prime}+1$ : update

$$
\pi_{j}^{i}\left(l^{\prime}+1\right)=\left\{\begin{array}{c}
\frac{S_{j}}{\bar{D}_{j}\left(l^{\prime}\right)} \text { if } j \in \mathcal{C}\left(l^{\prime}\right) \\
\Omega^{i}\left(l^{\prime}\right) \text { if } j \in \overline{\mathcal{C}}\left(l^{\prime}\right)
\end{array} .\right.
$$

4. Stop if $\left|\pi_{j}^{i}\left(l^{\prime}+1\right)-\pi_{j}^{i}\left(l^{\prime}\right)\right|<10^{-6}, \forall i=1 \ldots N, \forall j \in \mathcal{J}\left(l^{\prime}=\infty\right)$. The solution is: $D_{j}=\hat{D}_{j}\left(l^{\prime}\right)=\hat{D}_{j}(\infty), j \in \mathcal{J}, \mathcal{C}=\mathcal{C}\left(l^{\prime}\right)=\mathcal{C}(\infty), \Omega^{i}=\Omega^{i}\left(l^{\prime}\right)=\Omega^{i}(\infty)$ and $\bar{\Omega}_{j}=\bar{\Omega}_{j}\left(l^{\prime}\right)=\bar{\Omega}_{j}(\infty)$; else go to step 5 .

5. Update the MNL estimates with updated $\pi_{j}^{i}\left(l^{\prime}+1\right)$ among the explanatory variables in $\tilde{V}_{j}^{i}$. This gives $\tilde{\mathbb{P}}_{j}^{i}\left(l^{\prime}+1\right)=\frac{\exp \left(\tilde{V}_{j}^{i}\left(l^{\prime}+1\right)\right)}{\sum_{k \in \mathcal{J}} \exp \left(\tilde{V}_{k}^{i}\left(l^{\prime}+1\right)\right)}, \hat{\mathbb{P}}_{j}^{i}\left(l^{\prime}+1\right)=\frac{\tilde{\mathbb{P}}_{j}^{i}\left(l^{\prime}+1\right)}{\pi_{j}^{i}\left(l^{\prime}+1\right)}$ and $\hat{D}_{j}\left(l^{\prime}+1\right)=$ $\sum_{i=1}^{N} \hat{\mathbb{P}}_{j}^{i}\left(l^{\prime}+1\right)$

(a) Iteration $l=0$ (initialization): Compute the $\operatorname{set} \mathcal{C}\left(l^{\prime}+1,0\right)=\left\{j \in \mathcal{J} \mid S_{j} \leq \hat{D}_{j}\left(l^{\prime}+1\right)\right\}$ of alternatives constrained ex ante for demand at step $\left(l^{\prime}+1\right)$. 
(b) Compute the individual allocation ratio

$$
\Omega^{i}\left(l^{\prime}+1,0\right)=\left(1-\sum_{j \in \mathcal{C}\left(l^{\prime}+1,0\right)} \frac{S_{j}}{\hat{D}_{j}\left(l^{\prime}+1\right)} \hat{\mathbb{P}}_{j}^{i}\left(l^{\prime}+1\right)\right) /\left(1-\sum_{j \in \mathcal{C}\left(l^{\prime}+1,0\right)} \hat{\mathbb{P}}_{j}^{i}\left(l^{\prime}+1\right)\right) .
$$

(c) Compute the alternative-specific allocation ratio $\bar{\Omega}_{j}\left(l^{\prime}+1,0\right)=\frac{\sum_{i=1}^{N} \Omega^{i}\left(l^{\prime}+1,0\right) \hat{\mathbb{P}}_{j}^{i}\left(l^{\prime}+1\right)}{\sum_{i=1}^{N} \hat{\mathbb{P}}_{j}^{i}\left(l^{\prime}+1\right)}$.

(d) Update iteration of Algorithm K: $l \rightarrow l+1$. Update $\mathcal{C}\left(l^{\prime}+1, l+1\right)=\left\{j \in \mathcal{J} \mid S_{j} \leq \bar{\Omega}_{j}\left(l^{\prime}+1, l\right) \hat{D}_{j}\left(l^{\prime}+1\right)=\sum_{i=1}^{N} \Omega^{i}\left(l^{\prime}+1, l\right) \hat{\mathbb{P}}_{j}^{i}\left(l^{\prime}+1\right)\right\}$.

(e) Update $\Omega^{i}\left(l^{\prime}+1, l+1\right)=\frac{1-\sum_{j \in \mathcal{C}\left(l^{\prime}+1, l+1\right)} \frac{S_{j}}{\hat{D}_{j}\left(l^{\prime}+1\right)} \hat{\mathbb{P}}_{j}^{i}\left(l^{\prime}+1\right)}{1-\sum_{j \in \mathcal{C}\left(l^{\prime}+1, l+1\right)} \hat{\mathbb{P}}_{j}^{i}\left(l^{\prime}+1\right)}$.

(f) Update $\bar{\Omega}_{j}\left(l^{\prime}+1, l+1\right)=\frac{\sum_{i=1}^{N} \Omega^{i}\left(l^{\prime}+1, l+1\right) \hat{\mathbb{P}}_{j}^{i}\left(l^{\prime}+1\right)}{\sum_{i=1}^{N} \hat{\mathbb{P}}_{j}^{i}\left(l^{\prime}+1\right)}$.

(g) Stop Algorithm K at iteration $l+1$ if $\mathcal{C}\left(l^{\prime}+1, l+1\right)=\mathcal{C}\left(l^{\prime}+1, l\right)$ (and therefore $\Omega^{i}\left(l^{\prime}+1, l+1\right)=\Omega^{i}\left(l^{\prime}+1, l\right)$ and $\left.\bar{\Omega}_{j}\left(l^{\prime}+1, l+1\right)=\bar{\Omega}_{j}\left(l^{\prime}+1, l\right)\right)$, else go to step d.

(h) When convergence of Algorithm $\mathrm{K}$ is attained $(l=\infty)$, we have

$$
\begin{aligned}
& \mathcal{C}\left(l^{\prime}+1\right)=\left\{j \in \mathcal{J} \mid S_{j} \leq \bar{\Omega}_{j}\left(l^{\prime}+1, \infty\right) \hat{D}_{j}\left(l^{\prime}+1\right)\right\}, \Omega^{i}\left(l^{\prime}+1\right)=\Omega^{i}\left(l^{\prime}+1, \infty\right), \text { and } \\
& \bar{\Omega}_{j}\left(l^{\prime}+1\right)=\bar{\Omega}_{j}\left(l^{\prime}+1, \infty\right) .
\end{aligned}
$$

6. Go to step 3. 